\title{
Comparing homologies: Čech's theory, singular chains, integral flat chains and integral currents
}

Thierry De Pauw

\begin{abstract}
We give a new proof of a Theorem of S. Mardešić, generalized by G.E. Bredon, that Cech and singular homology groups of certain locally connected spaces coincide. We use the chain complexes of integral flat chains (H. Whitney) and integral currents (H. Federer and W.H. Fleming) to define new homology groups of subsets of Euclidean space. We show these verify the axioms of Eilenberg and Steenrod, and we provide Lipschitz-flavored local connectedness conditions which guarantee these groups coincide with Čech's. Relations between these theories is relevant for the solvability and regularity of many geometric variational problems.
\end{abstract}

\section{Foreword}

This paper is mostly devoted to comparing various homology groups of certain topological spaces $X$ (in the second part we will assume $X \subset \mathbb{R}^{n}$ ). The results proved here will be used in [6] in connection with the Plateau problem, that is the study of $m$ dimensional surfaces of least area bounded by a given $m-1$ dimensional cycle. This problem has received many distinct formulations. We start by briefly reviewing the account of E. R. Reifenberg [16] and that of H. Federer and W. H. Fleming [12]. For simplicity we restrict ourselves to the case when $m=2$ and the given boundary cycle is a simple closed curve $B \subset \mathbb{R}^{n}$. According to J.F. Adams (appendix of E. R. Reifenberg's paper op cit) a compact set $X \supset B$ is bounded by $B$ whenever the inclusion $B \rightarrow X$ induces a trivial homomorphism in homology

2000 Mathematics Subject Classification: 49Q15, 55N35, 55N40, 58A25.

Keywords: Čech homology, singular homology, locally connected spaces, integral flat chains, integral currents 
$H_{1}(B ; G) \rightarrow H_{1}(X ; G)$. For the purpose of proving the existence of a least area set $X$ (here area is understood as the 2 dimensional Hausdorff measure of $X$ ), the homology theory used in the definition needs to enjoy some stability property in the limit of a minimizing sequence. Cech's homology $\check{H}_{1}(\cdot ; G)$ with respect to a compact group $G$ of coefficients is appropriate in this setting. On the other hand H. Federer and W. H. Fleming assume that both $B$ and $X$ are sufficiently regular to be viewed as currents in the sense of G. de Rham. In that context there is a boundary operator defined as the dual of exterior differentiation and one asks for $B=\partial X$. Now if $X$ is an area minimizing surface in one of these settings, one would like to know if it is a proper competitor in the other. This amounts to comparing the Cech homology group $\check{H}_{1}(X ; G)$ with the homology group of $X$ obtained from the chain complex of (rectifiable) currents supported in $X$. For general dimension $m$ the classical Eilenberg-Steenrod Theorem does not apply to this question, for instance because one doesn't even know whether $X$ is triangulable. This paper is about finding local regularity conditions on a set $X$ (as opposed to combinatorial conditions) which allow for comparing various homology groups of $X$. We refer to [10] for a Definition of the singular homology $H_{q}(X ; \mathbb{Z})$ and the Čech homology $\check{H}_{q}(X ; \mathbb{Z})$ groups, as well as for a Definition of the simplicial homology group $H(K ; \mathbb{Z})$ of a simplicial complex $K$. For notational simplicity we restrict ourselves in $\mathbb{Z}$.

We start with the case when $X$ is a compact $C^{\infty}$ manifold without boundary. As a motivational discussion we briefly recall Weil's proof of a Theorem of de Rham (see [19]). The de Rham cohomology group $H_{D R}^{q}(X)$ is obtained from the cochain complex

$$
\cdots \stackrel{d}{\longrightarrow} \Omega^{q-1}(X) \stackrel{d}{\longrightarrow} \Omega^{q}(X) \stackrel{d}{\longrightarrow} \Omega^{q+1}(X) \stackrel{d}{\longrightarrow} \cdots
$$

where $\Omega^{q}(X)$ denotes the real vectorspace of smooth differential forms of degree $q$ on $X$, and $d$ is the exterior derivative. From the smoothness of $X$ we first infer the existence of a good open cover $\mathcal{U}=\left(U_{i}\right)_{i=0, \ldots, P}$. By good we mean that for each $p=0, \ldots, P$ and each $0 \leq i_{0}<\ldots<i_{p} \leq P$, the set $U_{i_{0}} \cap \ldots \cap U_{i_{p}}$, if nonempty, is diffeomorphic to a Euclidean space (it suffices to use a finite cover by normal neighborhoods with respect to some Riemannian metric, see e.g. [4, Theorem 5.1]). Next we associate with the open cover $\mathcal{U}$ a simplicial complex ([10, Chapter II $]) N(\mathcal{U})$ called its nerve: to each $U_{i}$ corresponds a vertex of $N(\mathcal{U})$; there is a 1-simplex in $N(\mathcal{U})$ with vertices $U_{i_{1}}$ and $U_{i_{2}}$ if and only if $U_{i_{1}} \cap U_{i_{2}} \neq \emptyset$; there is a 2-simplex in $N(\mathcal{U})$ with vertices $U_{i_{1}}, U_{i_{2}}$ and $U_{i_{3}}$ if and only if $U_{i_{1}} \cap U_{i_{2}} \cap U_{i_{3}} \neq \emptyset$; and so on. We are now in a position to state a version of the Theorem of de Rham: the simplicial cohomology group $H^{q}(N(\mathcal{U}) ; \mathbb{R})$ is isomorphic to $H_{D R}^{q}(X)$. In order to sketch a proof we consider the augmented double complex de- 


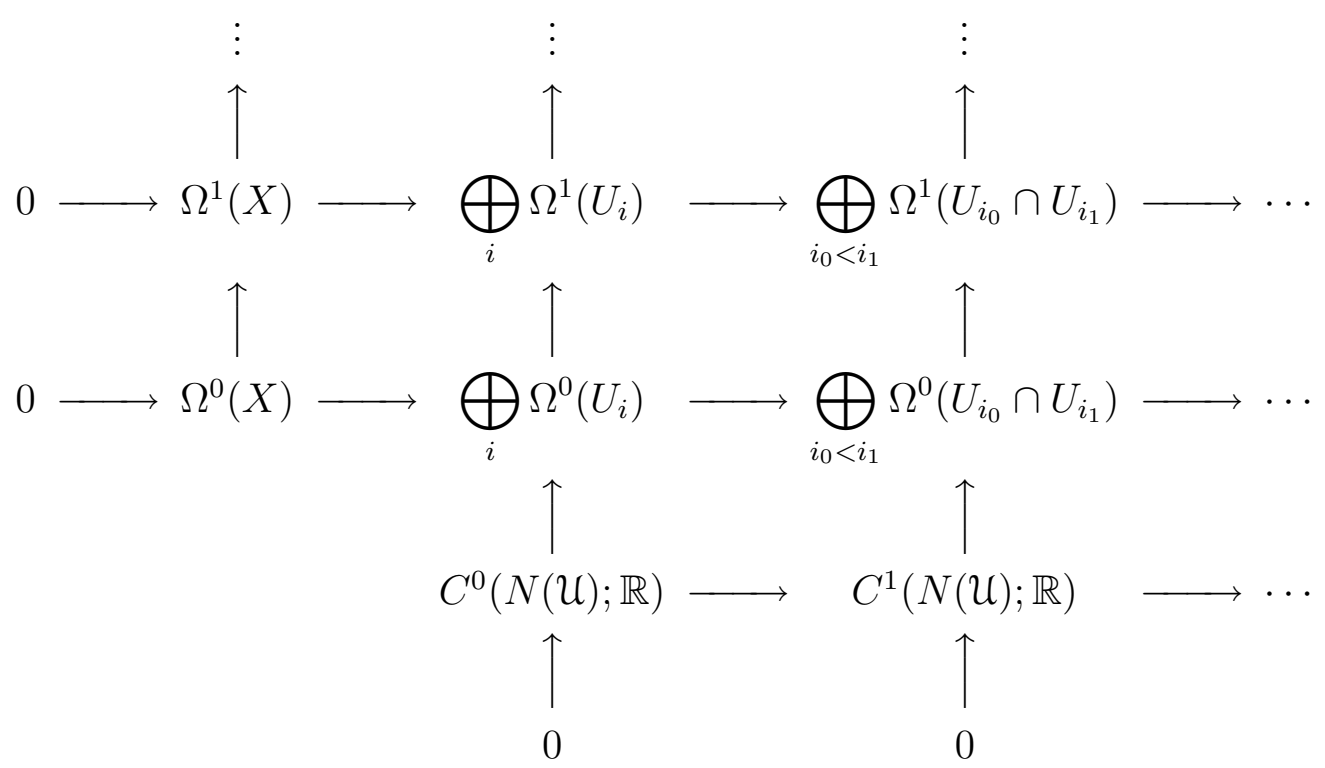

Figure 1: The Čech - de Rham complex.

picted on Figure 1, called the Cech-de Rham complex. The rows of this double complex are exact (the horizontal arrows are "restrictions of differential forms to open subsets" and exactness is checked by using partitions of unity, see [4, Proposition 8.5]). The columns are also exact according to our assumption that $\mathcal{U}$ is a good cover (recall that the converse of Poincaré's Lemma holds in $U_{i_{0}} \cap \ldots \cap U_{i_{p}}$, i.e. the reduced cohomology groups $H_{D R}^{*}\left(\mathbb{R}^{\nu}\right)$ are trivial). Now some diagram chasing (see e.g. Proposition 2.1 for a dual version) shows that $H^{*}(N(\mathcal{U}) ; \mathbb{R}) \cong H_{D R}^{*}(X)$.

Our main interest will be in homology rather than cohomology, which amounts to dualizing the diagram in Figure 1. Suppose that for each open set $U \subset X$ we are given "homology groups" $\mathfrak{H}_{q}(U)$ (say with coefficients in $\mathbb{Z}$ in the sense of [10, Chapter I $\S 6]$ ) which are associated with some chain complex, and denote by $\mathfrak{C}_{q}(U)$ the corresponding groups of " $q$-chains". Given an open cover $\mathcal{U}=\left(U_{i}\right)_{i}$ of $X$ (together with a total order on the index set) we consider the double complex associated with $\mathcal{U}$ and $\mathfrak{C}_{*}$, depicted on Figure 2. As before, in order to conclude that $H_{*}(N(\mathcal{U}) ; \mathbb{Z}) \cong \mathfrak{H}_{*}(X)$ it suffices to show that:

(A) The rows of the double complex in Figure 2 are exact;

(B) The columns of the double complex in Figure 2 are exact.

Suppose for instance that $\mathfrak{C}_{q}(U)$ are the groups of singular $q$-chains in $U$ 


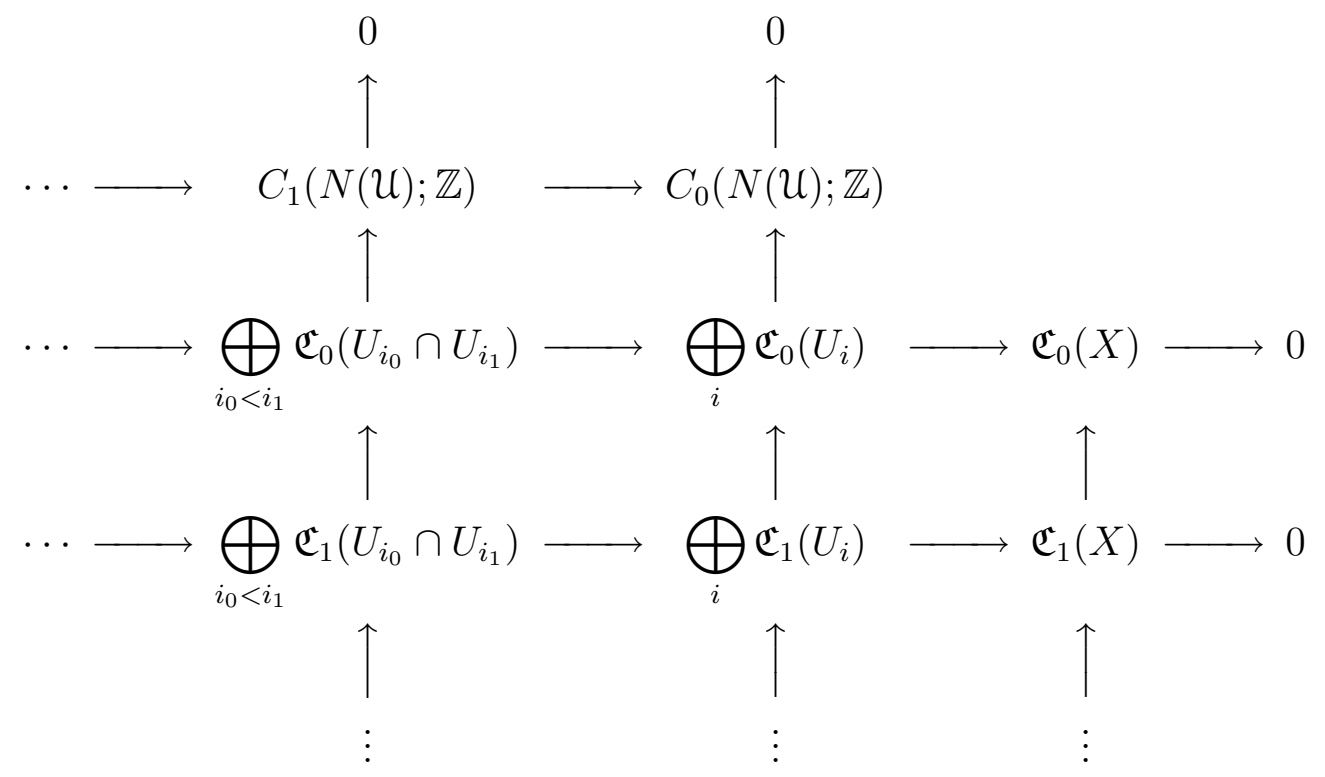

Figure 2: The double complex associated with $\mathcal{U}$ and $\mathfrak{C}_{*}$.

with coefficients in $\mathbb{Z}$. Exactness of the rows fails, but not for a good reason, and using barycentric subdivision one can define new groups of "chains" which yield the same homology groups and for which rows become exact (see Remark 2.11). We now specialize the notion of a good cover. We say that $\mathcal{U}$ is good relative to the homology theory $\mathfrak{H}$ (abbreviated as $\mathcal{U}$ is $\mathfrak{H}$-good) if either $U_{i_{0}} \cap \ldots \cap U_{i_{p}}=\emptyset$ or $\mathfrak{H}_{*}\left(U_{i_{0}} \cap \ldots \cap U_{i_{p}}\right) \cong\{0\}$ (reduced homology). In case when $\mathfrak{H}$ is singular homology and $X$ is a smooth compact submanifold of $\mathbb{R}^{n}$, a good cover of $X$ is clearly $\mathfrak{H}$-good as well, therefore the argument sketched above yields $\mathfrak{H}_{*}(X ; \mathbb{Z}) \cong H_{*}(N(\mathcal{U}) ; \mathbb{Z})$. If $X$ is merely $C^{1}$ one can refer to H. Whitney's Theorem that $X$ is homeomorphic to a smooth manifold and repeat the argument. If $X$ is a topological manifold however, it need not be homeomorphic to a smooth manifold and the existence of an $\mathfrak{H}$-good cover becomes problematic. Nevertheless for each $x \in X$ and each neighborhood $V \subset X$ of $x$ there exists an open set $U$ such that $x \in U \subset V$ and $\mathfrak{H}_{*}(U ; \mathbb{Z}) \cong\{0\}$ (reduced homology): one can choose $U$ to be homeomorphic to some Euclidean space. We will refer to this property by saying that $X$ is infinitesimally $\mathfrak{H}$-acyclic and we now investigate its consequences pertaining to the comparison of homology groups. Assume as before that the homology theory $\mathfrak{H}$ is obtained from some chain complex $\mathfrak{C}$ and let $\mathcal{U}$ be a cover of $X$. We suppose that the rows of the double complex on Figure 2 are exact (see Remark 2.11 for singular homology and Corollary 3.10 for homology groups computed from integral flat chains or integral currents) - this property is 
usually referred to by saying that $\mathfrak{C}$ is a cosheaf. The columns of the double complex, however, need not be exact and therefore we are unable to infer that $\mathfrak{H}_{*}(X ; \mathbb{Z}) \cong H_{*}(N(\mathcal{U}) ; \mathbb{Z})$. Nevertheless referring to the infinitesimal $\mathfrak{H}$-acyclicity of $X$ one can show (in case $X$ is paracompact) that the cover $\mathcal{U}$ may be refined to a cover $\mathcal{W}$ with the following property. For each $W \in \mathcal{W}$ there exists $u(W) \in \mathcal{U}$ such that $W \subset u(W)$ and if $W_{0}, \ldots, W_{p} \in \mathcal{W}$ either $W_{0} \cap \ldots \cap W_{p}=\emptyset$ or $\mathfrak{H}\left(W_{0} \cap \ldots \cap W_{p}\right) \rightarrow \mathfrak{H}\left(u\left(W_{0}\right) \cap \ldots \cap u\left(W_{p}\right)\right)$ is trivial (see [5, Theorem 4.4] and also Proposition 2.8). The columns of the double complex associated with the cover $\mathcal{W}$ are not exact either, yet a (columnwise) cycle "at the level" of $\mathcal{W}$ becomes a (column-wise) boundary when pushed forward "to the level" of $\mathcal{U}$. Playing some diagram chasing built on this observation now shows that the reduced homology groups $\mathfrak{H}_{*}(X ; \mathbb{Z})$ are isomorphic to the inverse limits

$$
\lim _{U \in \operatorname{Cov}(X)} H_{*}(N(\mathcal{U}) ; \mathbb{Z})
$$

with respect to all open covers of $X$. These are precisely the Cech homology groups $\check{H}_{*}(X ; \mathbb{Z})$; in particular $\check{H}_{*}(X ; \mathbb{Z}) \cong H_{*}(X ; \mathbb{Z})$ (the latter is singular homology). This result was obtained by S. Mardešić (see [13]). G.E. Bredon generalized it to homology with coefficients in a cosheaf (see [5]). The proof we present in this paper is different and perhaps more elementary than Bredon's (in that we avoid the use of spectral sequences) and more general than Mardešić's (in that we aren't restricted to integral coefficients). We hope the able reader will accept our apologies for providing too many details.

We close this paper with an introduction to integral currents and integral flat chains ${ }^{1}$. We show that homology groups corresponding to the chain complexes of integral currents (resp. integral flat chains) form a homology theory in the sense of Eilenberg and Steenrod (compare with [11, 4.4.1] for a weaker statement). The following ensues from the general comparison Theorem presented above: if $X \subset \mathbb{R}^{n}$ is a Lipschitzian manifold then the Cech homology groups (with integral coefficients) of $X$ and the homology groups of $X$ relative to integral currents (resp. integral flat chains) coincide (note that Lipschitzian manifolds need not be triangulable, see e.g. [18]). The condition that $X$ be Lipschitzian can be weakened to various other "infinitesimal acyclicity" conditions. That these conditions must differ according to whether we deal with integral currents or integral flat chains is illustrated by various examples in the last subsection.

We finish this introduction by briefly sketching the content of each part of the paper. Section 2 starts with the necessary definition of chain com-

\footnotetext{
${ }^{1}$ Flat chains (with real coefficients) were introduced by H. Whitney in [20] whereas integral currents first appeared in H. Federer and W.H. Fleming's paper [12].
} 
plexes and double complexes. Proposition 2.1 is the (dualized) core of Weil's proof of de Rham's Theorem, whereas Theorems 2.3 and 2.4 constitute an improvement on this Proposition needed for our main result. Subsection 2.2 briefly surveys Cech homology and the concept of a cosheaf. The main result (comparing Čech and singular homology) is Theorem 2.15. Subsection 3.1 and 3.2 survey integral currents and integral flat chains respectively. Subsection 3.3 contains the Definition of homology groups of $X \subset \mathbb{R}^{n}$ obtained from the chain complexes of integral currents and integral flat chains respectively. The slicing Lemma 3.5 is the main tool for showing these homology theories verify the Excision Axiom (Proposition 3.7) and act as a cosheaf on the category $\mathrm{O}(X)$ of open subsets of $X \subset \mathbb{R}^{n}$ (Corollary 3.10). The main result for comparing these homology theories to Čech's is Theorem 3.14. Finally subsection 3.4 contains elementary examples showing the four homology theories considered here do not coincide in general. Appendix A contains vocabulary from category theory and Appendix B gathers some vocabulary from geometric measure theory.

It is the author's pleasure to express many thanks to his colleagues Y. Félix and P. Lambrechts for their help and patience.

\section{A general comparison Theorem}

\section{1. (Double) chain complexes}

In this paper all groups are abelian. A chain complex consists of an indexed family of groups $A_{q}, q=0,1,2, \ldots$, together with homomorphisms $\Psi_{q}$ : $A_{q} \rightarrow A_{q-1}, q=1,2, \ldots$, verifying the additional requirement that im $\Psi_{q+1} \subset$ ker $\Psi_{q}, q=1,2, \ldots$. We will usually refer to a chain complex using the notation $\left(A_{*} ; \Psi_{*}\right)$, or simply $A_{*}$ when the homomorphisms $\Psi_{*}$ are clearly identified by the context. The homology groups of $A_{*}$ are defined by

$$
H_{q}\left(A_{*}\right)=\frac{\operatorname{ker} \Psi_{q}}{\operatorname{im} \Psi_{q+1}}, \quad q=1,2, \ldots, \quad \text { and } \quad H_{0}\left(A_{*}\right)=\frac{A_{0}}{\operatorname{im} \Psi_{1}} .
$$

A morphism of chain complexes $f: A_{*} \rightarrow A_{*}^{\prime}$ consists of an indexed family of homomorphisms $f_{q}: A_{q} \rightarrow A_{q}^{\prime}$ such that $f_{q} \circ \Psi_{q+1}=\Psi_{q+1}^{\prime} \circ f_{q+1}$, $q=0,1,2, \ldots$. This condition implies that $f$ induces homomorphisms in homology, $H_{q}(f): H_{q}\left(A_{*}\right) \rightarrow H_{q}\left(A_{*}^{\prime}\right)$, well-defined by

$$
H_{q}(f)([a])=\left[f_{q}(a)\right], \quad q=0,1,2, \ldots
$$

Whenever $A_{*} \stackrel{f}{\rightarrow} A_{*}^{\prime} \stackrel{g}{\rightarrow} A_{*}^{\prime \prime}$ are morphisms of chain complexes we define a morphism of chain complexes $g \circ f: A_{*} \rightarrow A_{*}^{\prime \prime}$ by the formula $(g \circ f)_{q}=g_{q} \circ f_{q}$, $q=0,1,2, \ldots$, and we notice that $H_{q}(g \circ f)=H_{q}(g) \circ H_{q}(f)$. 
A double chain complex consists of an indexed family of groups $A_{p, q}$ together with homomorphisms

$$
\Phi_{p+1, q}: A_{p+1, q} \rightarrow A_{p, q} \quad \text { and } \quad \Psi_{p, q+1}: A_{p, q+1} \rightarrow A_{p, q}, \quad p, q=0,1,2, \ldots,
$$

verifying the following conditions:

(A) for each $q=0,1,2, \ldots,\left(A_{*, q} ; \Phi_{*, q}\right)$ is a chain complex;

(B) for each $p=0,1,2, \ldots,\left(A_{p, *} ; \Psi_{p, *}\right)$ is a chain complex;

(C) $\Psi_{p, q+1} \circ \Phi_{p+1, q+1}=\Phi_{p+1, q} \circ \Psi_{p+1, q+1}$ whenever $p, q=0,1,2, \ldots$

We will refer to such a double chain complex using the notation $\left(A_{*, *} ; \Phi_{*, *}\right.$; $\left.\Psi_{*, *}\right)$, or simply $A_{*, *}$ when the homomorphisms $\Phi_{*, *}$ and $\Psi_{*, *}$ can be clearly identified by the context. With a double chain complex $\left(A_{*, *} ; \Phi_{*, *} ; \Psi_{*, *}\right)$ we associate a chain complex $\left(\mathcal{A}_{*} ; \Delta_{*}\right)$, called the total complex of $A_{*, *}$, defined as follows. We set

$$
\mathcal{A}_{m}=\bigoplus_{\substack{p, q=0, \ldots, m \\ p+q=m}} A_{p, q}, m=0,1,2, \ldots
$$

and for $a \in \mathcal{A}_{m}, m \geq 1$, we define

$$
\Delta_{m}(a)=\sum_{\substack{p \geq 1, q \geq 0 \\ p+q=m}}\left(z_{p-1, q} \circ \Phi_{p, q} \circ y_{p, q}\right)(a)+\sum_{\substack{p \geq 0, q \geq 1 \\ p+q=m}}(-1)^{p}\left(z_{p, q-1} \circ \Psi_{p, q} \circ y_{p, q}\right)(a)
$$

where $z_{p, q}: A_{p, q} \rightarrow \mathcal{A}_{m}$ and $y_{p, q}: \mathcal{A}_{m} \rightarrow A_{p, q}$ are the obvious inclusions and projections respectively. One trivially checks that $\Delta_{m+1} \circ \Delta_{m}=0$. The homology groups of the chain complex $\left(\mathcal{A}_{*} ; \Delta_{*}\right)$ will be denoted by $H_{m}\left(A_{*, *}\right)$, $m=0,1,2, \ldots$.

A morphism of double chain complexes

$$
f:\left(A_{*, *} ; \Phi_{*, *} ; \Psi_{*, *}\right) \rightarrow\left(A_{*, *}^{\prime} ; \Phi_{*, *}^{\prime} ; \Psi_{*, *}^{\prime}\right)
$$

consists of an indexed family of homomorphisms $f_{p, q}: A_{p, q} \rightarrow A_{p, q}^{\prime}, p, q=$ $0,1,2, \ldots$, such that:

(A) for every $q=0,1,2, \ldots, f_{*, q}: A_{*, q} \rightarrow A_{*, q}^{\prime}$ is a morphism of chain complexes;

(B) for every $p=0,1,2, \ldots, f_{p, *}: A_{p, *} \rightarrow A_{p, *}^{\prime}$ is a morphism of chain complexes.

One readily checks that $f$ induces a morphism of chain complexes $\bar{f}$ : $\left(\mathcal{A}_{*} ; \Delta_{*}\right) \rightarrow\left(\mathcal{A}_{*}^{\prime} ; \Delta_{*}^{\prime}\right)$ defined as follows:

$$
\bar{f}_{m}(a)=\sum_{\substack{p \geq 0, q \geq 0 \\ p+q=m}}\left(z_{p, q}^{\prime} \circ f_{p, q} \circ y_{p, q}\right)(a), a \in \mathcal{A}_{m} .
$$


An augmented double chain complex consists of the following data: a double chain complex $\left(A_{*, *} ; \Phi_{*, *} ; \Psi_{*, *}\right)$, an ordered pair of chain complexes $\left(L_{*} ; \Psi_{*}\right)$ and $\left(M_{*} ; \Phi_{*}\right)$ together with families of homomorphisms $\Phi_{0, q}: A_{0, q} \rightarrow L_{q}$, $q=0,1,2, \ldots$, and $\Psi_{p, 0}: A_{p, 0} \rightarrow M_{p}, p=0,1,2, \ldots$, verifying the following conditions:

(A) $\operatorname{im} \Phi_{1, q} \subset \operatorname{ker} \Phi_{0, q}$ and $\operatorname{im} \Psi_{p, 1} \subset \operatorname{ker} \Psi_{p, 0}$ for every $p, q=0,1,2, \ldots$;

(B) $\Psi_{q+1} \circ \Phi_{0, q+1}=\Phi_{0, q} \circ \Psi_{0, q+1}$ for every $q=0,1,2, \ldots$;

(C) $\Phi_{p+1} \circ \Psi_{p+1,0}=\Psi_{p, 0} \circ \Phi_{p+1,0}$ for every $p=0,1,2, \ldots$

One readily checks that, for each $m=0,1,2, \ldots, \Phi_{0, m} \circ y_{0, m}: \mathcal{A}_{m} \rightarrow L_{m}$ and $\Psi_{m, 0} \circ y_{m, 0}: \mathcal{A}_{m} \rightarrow M_{m}$ induce homomorphisms in homology:

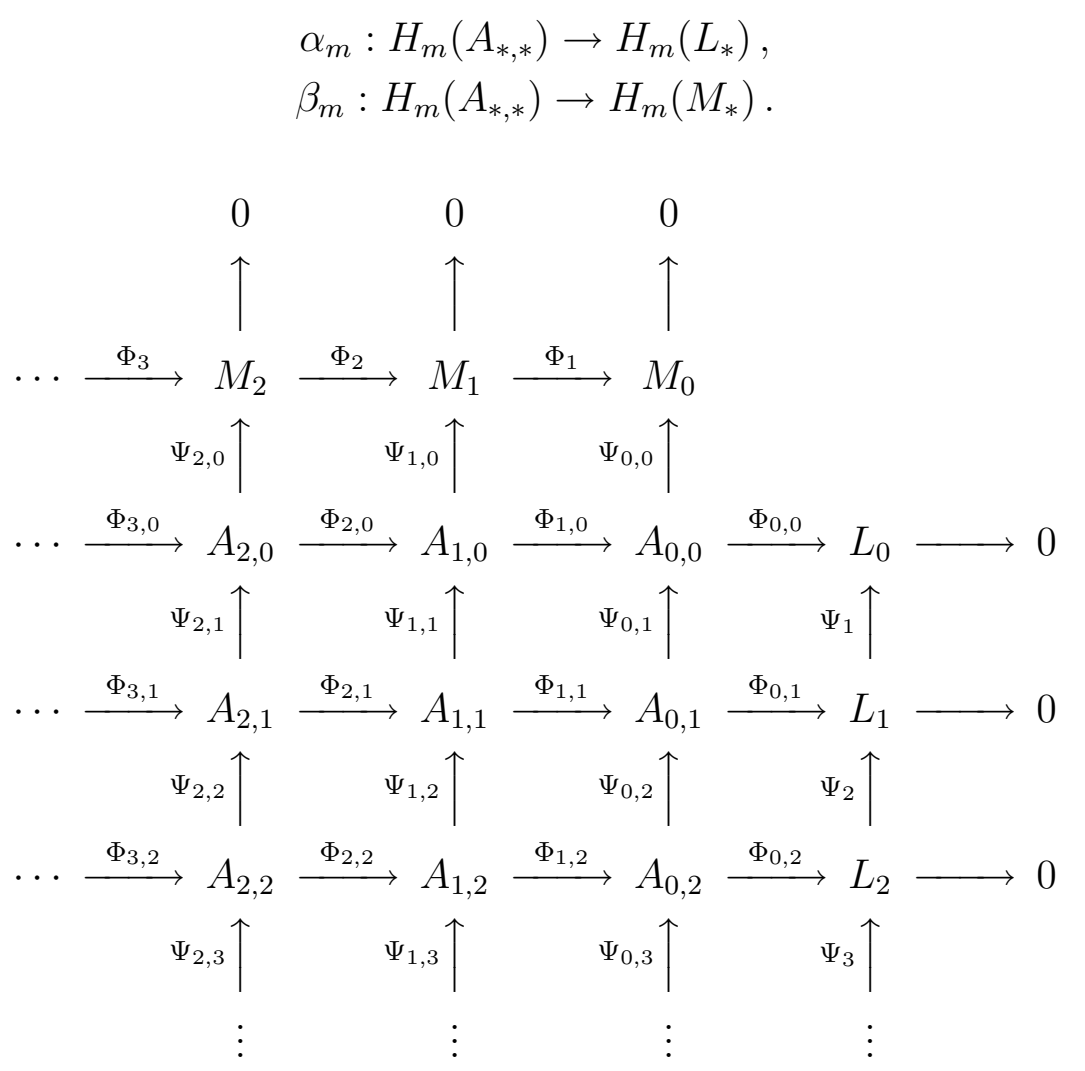

The following Proposition and its proof are classical.

Proposition 2.1. Let $A_{*, *}$ be a double chain complex augmented by the two chain complexes $L_{*}$ and $M_{*}$ as pictured on the diagram above, and let $m \geq 0$. Assume that for each $q=0, \ldots, m+1$ the corresponding line is exact:

$$
A_{m+1-q, q} \stackrel{\Phi_{m+1-q, q}}{\longrightarrow} \cdots \stackrel{\Phi_{2, q}}{\longrightarrow} A_{1, q} \stackrel{\Phi_{1, q}}{\longrightarrow} A_{0, q} \stackrel{\Phi_{0, q}}{\longrightarrow} L_{q} \longrightarrow 0 .
$$

Then $\alpha_{m}: H_{m}\left(A_{*, *}\right) \rightarrow H_{m}\left(L_{*}\right)$ is an isomorphism. 
Proof. We start by showing that $\alpha_{m}$ is surjective. Let $b \in L_{m}$ and assume that $\Psi_{m}(b)=0$ in case $m \geq 1$. Since the $m^{\text {th }}$ line is exact at $L_{m}$ there exists $a_{0, m} \in A_{0, m}$ such that $\Phi_{0, m}\left(a_{0, m}\right)=b$. If $m=0$ this shows that $\alpha_{0}$ is onto; otherwise we will define inductively a sequence $a_{k, m-k} \in A_{k, m-k}$, $k=1, \ldots, m$, such that

$$
(-1)^{k} \Phi_{k, m-k}\left(a_{k, m-k}\right)+\Psi_{k-1, m-k+1}\left(a_{k-1, m-k+1}\right)=0 .
$$

For $k=1$ we notice that

$$
\Phi_{0, m-1}\left(\Psi_{0, m}\left(a_{0, m}\right)\right)=\Psi_{m}\left(\Phi_{0, m}\left(a_{0, m}\right)\right)=\Psi_{m}(b)=0 .
$$

Since the $(m-1)^{t h}$ line is exact at $A_{0, m-1}$ there exists $a_{1, m-1} \in A_{1, m-1}$ such that $\Phi_{1, m-1}\left(a_{1, m-1}\right)=\Psi_{0, m}\left(a_{0, m}\right)$ so that $(2.1)$ is verified for $k=1$. Assume now it is verified for $k-1$ and observe that

$$
\begin{aligned}
\Phi_{k-1, m-k} & \left(\Psi_{k-1, m-k+1}\left(a_{k-1, m-k+1}\right)\right) \\
& =\Psi_{k-2, m-k+1}\left(\Phi_{k-1, m-k+1}\left(a_{k-1, m-k+1}\right)\right) \\
& =(-1)^{k} \Psi_{k-2, m-k+1}\left(\Psi_{k-2, m-k+2}\left(a_{k-2, m-k+2}\right)\right) \\
& =0 .
\end{aligned}
$$

Since the $(m-k)^{t h}$ line is exact at $A_{k-1, m-k}$ there exists $a_{k, m-k} \in A_{k, m-k}$ such that (2.1) is verified. Letting

$$
a=\sum_{k=0}^{m} z_{k, m-k}\left(a_{k, m-k}\right)
$$

we infer from (2.1) that $\Delta_{m}(a)=0$. On the other hand the choice of $a_{0, m}$ ensures that $\alpha_{m}([a])=[b]$, showing that $\alpha_{m}$ is onto.

We now turn to checking that $\alpha_{m}$ is injective. Let $a \in \mathcal{A}_{m}$ be such that $\Phi_{0, m}\left(a_{0, m}\right)=\Psi_{m+1}(b)$ for some $b \in L_{m+1}$, where we have set $a_{k, m-k}=$ $y_{k, m-k}(a), k=0, \ldots, m$. Since the $(m+1)^{t h}$ line is exact at $L_{m+1}$ there exists $c_{0, m+1} \in A_{0, m+1}$ such that $\Phi_{0, m+1}\left(c_{0, m+1}\right)=b$. We will define inductively a sequence $c_{k, m+1-k} \in A_{k, m+1-k}, k=1, \ldots, m+1$, such that

$$
\Phi_{k, m+1-k}\left(c_{k, m+1-k}\right)+(-1)^{k-1} \Psi_{k-1, m+2-k}\left(c_{k-1, m+2-k}\right)=a_{k-1, m+1-k} .
$$

For $k=1$ we observe that

$$
\Phi_{0, m}\left(\Psi_{0, m+1}\left(c_{0, m+1}\right)-a_{0, m}\right)=\Psi_{m+1}\left(\Phi_{0, m+1}\left(c_{0, m+1}\right)\right)-\Psi_{m+1}(b)=0 .
$$

Since the $m^{\text {th }}$ line is exact at $A_{0, m}$ there exists $c_{1, m} \in A_{1, m}$ so that $\Phi_{1, m}\left(c_{1, m}\right)=$ $-\Psi_{0, m+1}\left(c_{0, m+1}\right)+a_{0, m}$, whence $(2.2)$ is verified for $k=1$. 
We now assume $m \geq 1, \Delta_{m}(a)=0$ and (2.2) is verified for $k-1$. We notice that

$$
\begin{aligned}
& \Phi_{k-1, m+1-k}\left((-1)^{k-1} \Psi_{k-1, m+2-k}\left(c_{k-1, m+2-k}\right)-a_{k-1, m+1-k}\right) \\
& =(-1)^{k-1} \Psi_{k-2, m+2-k}\left(\Phi_{k-1, m+2-k}\left(c_{k-1, m+2-k}\right)\right) \\
& \quad-\Phi_{k-1, m+1-k}\left(a_{k-1, m+1-k}\right) \\
& =(-1)^{k-1} \Psi_{k-2, m+2-k}\left(a_{k-2, m+2-k}-(-1)^{k-2} \Psi_{k-2, m+3-k}\left(c_{k-2, m+3-k}\right)\right) \\
& \quad-\Phi_{k-1, m+1-k}\left(a_{k-1, m+1-k}\right) \\
& =-y_{k-2, m+1-k}\left(\Delta_{m}(a)\right) \\
& =0
\end{aligned}
$$

Since the $(m+1-k)^{t h}$ line is exact at $A_{k-1, m+1-k}$ there exists $c_{k, m+1-k} \in$ $A_{k, m+1-k}$ such that (2.2) is verified for $k$. Letting

$$
c=\sum_{k=0}^{m+1} z_{k, m+1-k}\left(c_{k, m+1-k}\right)
$$

we infer from (2.2) that $\Delta_{m+1}(c)=a$, showing that $\alpha_{m}$ is one-to-one.

Let $\left(A_{*, *}^{i} ; \Phi_{*, *}^{i} ; \Psi_{*, *}^{i}\right)$ be double chain complexes augmented by chain complexes $\left(L_{*}^{i} ; \Psi_{*}^{i}\right)$ and $\left(M_{*}^{i} ; \Phi_{*}^{i}\right), i=0,1$. A morphism of augmented double chain complexes $f:\left(A_{*, *}^{1} ; L_{*}^{1} ; M_{*}^{1}\right) \rightarrow\left(A_{*, *}^{0} ; L_{*}^{0} ; M_{*}^{0}\right)$ consists of the following data: indexed families of homomorphisms $f_{p, q}: A_{p, q}^{1} \rightarrow A_{p, q}^{0}$, $p, q=0,1,2, \ldots, f_{-1, q}: L_{q}^{1} \rightarrow L_{q}^{0}, q=0,1,2, \ldots$, and $f_{p,-1}: M_{p}^{1} \rightarrow M_{p}^{0}$, $p=0,1,2, \ldots$, verifying the following conditions:

(A) the restriction $f: A_{*, *}^{1} \rightarrow A_{*, *}^{0}$ is a morphism of double chain complexes;

(B) $f_{-1, *}: L_{*}^{1} \rightarrow L_{*}^{0}$ and $f_{*,-1}: M_{*}^{1} \rightarrow M_{*}^{0}$ are morphisms of chain complexes;

(C) $f_{-1, q} \circ \Phi_{0, q}^{1}=\Phi_{0, q}^{0} \circ f_{0, q}$ for every $q=0,1,2, \ldots$;

(D) $f_{p,-1} \circ \Psi_{p, 0}^{1}=\Psi_{p, 0}^{0} \circ f_{p, 0}$ for every $p=0,1,2, \ldots$.

We will often consider the case when the chain complexes $L_{*}^{1}$ and $L_{*}^{0}$ coincide - it will then be implicitely assumed that $f_{-1, *}$ is the identity.

For further reference we state explicitely the following rather obvious result.

Lemma 2.2. Let $\left(A_{*, *}^{i} ; L_{*}^{i} ; M_{*}^{i}\right), i=0,1$, be augmented double chain complexes, and let $f:\left(A_{*, *}^{1} ; L_{*}^{1} ; M_{*}^{1}\right) \rightarrow\left(A_{*, *}^{0} ; L_{*}^{0} ; M_{*}^{0}\right)$ be a morphism of augmented double chain complexes. Then $H_{m}\left(f_{-1, *}\right) \circ \alpha_{m}^{1}=\alpha_{m}^{0} \circ H_{m}(\bar{f})$ and $H_{m}\left(f_{*,-1}\right) \circ \beta_{m}^{1}=\beta_{m}^{0} \circ H_{m}(\bar{f})$ for each $m=0,1,2, \ldots$. 
We are now ready for the main technical statement of this section. Hypothesis (5) below should be understood as a way around the lack of vertical exactness of the augmented double chain complex $\left(A_{*, *}^{0} ; L_{*}^{0} ; M_{*}^{0}\right)$.

Theorem 2.3. Assume that:

(1) $m \geq 1$ is an integer;

(2) $\left(\left(A_{*, *}^{j} ; \Phi_{*, *}^{j} ; \Psi_{*, *}^{j}\right) ;\left(L_{*} ; \Psi_{*}\right) ;\left(M_{*}^{j} ; \Phi_{*}^{j}\right)\right), j=0, \ldots, m+1$, are augmented double chain complexes (notice that $\left(L_{*} ; \Psi_{*}\right)$ doesn't depend upon $\left.j\right)$;

(3) for every $j=0, \ldots, m+1$ and every $q=0, \ldots, m+1$, the following sequence is exact:

$$
A_{m+1-q, q}^{j} \stackrel{\Phi_{m+1-q, q}^{j}}{\longrightarrow} \cdots \stackrel{\Phi_{2, q}^{j}}{\longrightarrow} A_{1, q}^{j} \stackrel{\Phi_{1, q}^{j}}{\longrightarrow} A_{0, q}^{j} \stackrel{\Phi_{0, q}^{j}}{\longrightarrow} L_{q} \longrightarrow 0
$$

(4) for the pairs $(j, p)=(m+1, m+1)$ and $(j, p)=(m, m)$ the homomorphism $\Psi_{p, 0}^{j}$ is surjective;

(5) $\chi^{j}:\left(A_{*, *}^{j} ; L_{*} ; M_{*}^{j}\right) \rightarrow\left(A_{*, *}^{j-1} ; L_{*} ; M_{*}^{j-1}\right), j=1, \ldots, m+1$, are morphisms of augmented double chain complexes such that for every $p=$ $0, \ldots, m+1$ and every $q=0, \ldots, m+1-p$ the following holds:

$$
\chi_{p, q}^{j}\left(\operatorname{ker} \Psi_{p, q}^{j}\right) \subset \operatorname{im} \Psi_{p, q+1}^{j-1} .
$$

Then the following conclusions hold:

(6) for every $\left[b^{m}\right] \in H_{m}\left(M_{*}^{m}\right)$ there exists $\left[a^{0}\right] \in H_{m}\left(A_{*, *}^{0}\right)$ such that

$$
\beta_{m}^{0}\left(\left[a^{0}\right]\right)=H_{m}\left(\chi_{*,-1}^{1} \circ \cdots \circ \chi_{*,-1}^{m}\right)\left(\left[b^{m}\right]\right) ;
$$

(7) $\beta_{m}^{m+1}: H_{m}\left(A_{*, *}^{m+1}\right) \rightarrow H_{m}\left(M_{*}^{m+1}\right)$ is injective.

Proof. We start by proving conclusion (6). Let $b^{m} \in M_{m}^{m}$ be such that $\Phi_{m}^{m}\left(b^{m}\right)=0$. According to hypothesis (4) there exists $a_{m, 0}^{m} \in A_{m, 0}^{m}$ such that $\Psi_{m, 0}^{m}\left(a_{m, 0}^{m}\right)=b^{m}$. We will define inductively a sequence $a_{m-k, k}^{m-k} \in A_{m-k, k}^{m-k}$, $k=1, \ldots, m$, such that

$$
(-1)^{m-k} \Psi_{m-k, k}^{m-k}\left(a_{m-k, k}^{m-k}\right)+\Phi_{m-k+1, k-1}^{m-k}\left(a_{m-k+1, k-1}^{m-k}\right)=0
$$

where we have set

$$
a_{m-k+1, k-1}^{m-k}=\chi_{m-k+1, k-1}^{m-k+1}\left(a_{m-k+1, k-1}^{m-k+1}\right) .
$$

For $k=1$ we observe that

$$
\Psi_{m-1,0}^{m}\left(\Phi_{m, 0}^{m}\left(a_{m, 0}^{m}\right)\right)=\Phi_{m}^{m}\left(\Psi_{m, 0}^{m}\left(a_{m, 0}^{m}\right)\right)=0 .
$$


From hypothesis (5) we infer that

$$
\operatorname{im} \Psi_{m-1,1}^{m-1} \ni \chi_{m-1,0}^{m}\left(\Phi_{m, 0}^{m}\left(a_{m, 0}^{m}\right)\right)=\Phi_{m, 0}^{m-1}\left(a_{m, 0}^{m-1}\right),
$$

whence there exists $a_{m-1,1}^{m-1} \in A_{m-1,1}^{m-1}$ such that (2.3) is verified for $k=1$. We now assume that (2.3) is verified for $k-1$ and we check that

$$
\begin{aligned}
\Psi_{m-k, k-1}^{m-k+1}\left(\Phi_{m-k+1, k-1}^{m-k+1}\right. & \left.\left(a_{m-k+1, k-1}^{m-k+1}\right)\right)=\Phi_{m-k+1, k-2}^{m-k+1}\left(\Psi_{m-k+1, k-1}^{m-k+1}\left(a_{m-K+1, k-1}^{m-k+1}\right)\right) \\
= & (-1)^{m-k} \Phi_{m-k+1, k-2}^{m-k+1}\left(\Phi_{m-k+2, k-2}^{m-k+1}\left(a_{m-k+2, k-2}^{m-k+1}\right)\right) \\
= & 0 .
\end{aligned}
$$

According to hypothesis (5) again, we infer that

$$
\begin{aligned}
\operatorname{im} \Psi_{m-k, k}^{m-k} & \ni \chi_{m-k, k-1}^{m-k+1}\left(\Phi_{m-k+1, k-1}^{m-k+1}\left(a_{m-k+1, k-1}^{m-k+1}\right)\right) \\
& =\Phi_{m-k+1, k-1}^{m-k}\left(a_{m-k+1, k-1}^{m-k}\right) .
\end{aligned}
$$

Therefore (2.3) is verified for some $a_{m-k, k}^{m-k} \in A_{m-k, k}^{m-k}$. Next we define for each $k=0, \ldots, m$ :

$$
a_{m-k, k}^{0}=\left(\chi_{m-k, k}^{1} \circ \cdots \circ \chi_{m-k, k}^{m-k}\right)\left(a_{m-k, k}^{m-k}\right),
$$

and we let

$$
a^{0}=\sum_{k=0}^{m} z_{m-k, k}^{0}\left(a_{m-k, k}^{0}\right) .
$$

It is now transparent that $\Psi_{m, 0}^{0}\left(a_{m, 0}^{0}\right)=\left(\chi_{m,-1}^{1} \circ \cdots \circ \chi_{m,-1}^{m}\right)\left(b^{m}\right)$ and that (2.3) implies $\Delta_{m}^{0}\left(a^{0}\right)=0$. This proves (6).

We now turn to showing that $\beta_{m}^{m+1}$ is injective. Let $a \in \operatorname{ker} \Delta_{m}^{m+1}$ be such that $\Psi_{m, 0}^{m+1}\left(a_{m, 0}^{m+1}\right)=\Phi_{m+1}^{m+1}\left(b^{m+1}\right)$ for some $b \in M_{m+1}^{m+1}$, where we have put $a_{m-k, k}^{m+1}=y_{m-k, k}^{m+1}(a), k=0, \ldots, m$. According to hypothesis (4) there exists $c_{m+1,0}^{m+1} \in A_{m+1,0}^{m+1}$ such that $\Psi_{m+1,0}^{m+1}\left(c_{m+1,0}^{m+1}\right)=b$. We will define inductively a sequence $c_{m+1-k, k}^{m+1-k} \in A_{m+1-k, k}^{m+1-k}, k=1, \ldots, m+1$, such that

$$
\begin{aligned}
(-1)^{m+1-k} \Psi_{m+1-k, k}^{m+1-k}\left(c_{m+1-k, k}^{m+1-k}\right)+\Phi_{m+2-k, k-1}^{m+1-k}\left(c_{m+1-k, k-1}^{m+1-k}\right) & \\
= & a_{m+1-k, k-1}^{m+1-k}
\end{aligned}
$$

where we have put

$$
c_{m+2-k, k-1}^{m+1-k}=\chi_{m+2-k, k-1}^{m+2-k}\left(c_{m+2-k, k-1}^{m+2-k}\right),
$$

and

$$
a_{m-k, k}^{i}=\left(\chi_{m-k, k}^{i+1} \circ \cdots \circ \chi_{m-k, k}^{m+1}\right)\left(a_{m-k, k}^{m+1}\right), i=0, \ldots, m .
$$


For $k=1$ we observe that

$$
\Psi_{m, 0}^{m+1}\left(\Phi_{m+1,0}^{m+1}\left(c_{m+1,0}^{m+1}\right)-a_{m, 0}^{m+1}\right)=\Phi_{m+1}^{m+1}\left(\Psi_{m+1,0}^{m+1}\left(c_{m+1,0}^{m+1}\right)\right)-\Psi_{m, 0}^{m+1}\left(a_{m, 0}^{m+1}\right)=0 .
$$

It follows from hypothesis (5) that

$$
\begin{aligned}
\operatorname{im} \Psi_{m, 1}^{m} & \ni \chi_{m, 0}^{m+1}\left(\Phi_{m+1,0}^{m+1}\left(c_{m+1,0}^{m+1}\right)-a_{m, 0}^{m+1}\right) \\
& =\Phi_{m+1,0}^{m}\left(c_{m+1,0}^{m}\right)-a_{m, 0}^{m},
\end{aligned}
$$

which shows that (2.4) is verified when $k=1$. We now assume that (2.4) is verified for $k-1$ and we check that:

$$
\begin{aligned}
\Psi_{m+1-k, k-1}^{m+2-k} & \left(\Phi_{m+2-k, k-1}^{m+2-k}\left(c_{m+2-k, k-1}^{m+2-k}\right)-a_{m+1-k, k-1}^{m+2-k}\right) \\
= & \Phi_{m+2-k, k-2}^{m+2-k}\left(\Psi_{m+2-k, k-1}^{m+2-k}\left(c_{m+2-k, k-1}^{m+2-k}\right)\right)-\Psi_{m+1-k, k-1}^{m+2-k}\left(a_{m+1-k, k-1}^{m+2-k}\right) \\
= & (-1)^{m+2-k} \Phi_{m+2-k, k-2}^{m+2-k}\left(a_{m+2-k, k-2}^{m+2-k}-\Phi_{m+3-k, k-2}^{m+2-k}\left(c_{m+3-k, k-2}^{m+2-k}\right)\right) \\
& \quad \quad-\Psi_{m+1-k, k-1}^{m+2-k}\left(a_{m+1-k, k-1}^{m+2-k}\right) \\
= & -y_{m+1-k, k-1}^{m+2-k}\left(\Delta_{m}^{m+2-k}\left(a^{m+2-k}\right)\right) \\
= & 0 .
\end{aligned}
$$

Again according to hypothesis (5) it results that

$$
\begin{aligned}
\operatorname{im} \Psi_{m+1-k, k}^{m+1-k} & \ni \chi_{m+1-k, k-1}^{m+2-k}\left(\Phi_{m+2-k, k-1}^{m+2-k}\left(c_{m+2-k, k-1}^{m+2-k}\right)-a_{m+2-k, k-1}^{m+2-k}\right) \\
& =\Phi_{m+2-k, k-1}^{m+1-k}\left(c_{m+2-k, k-1}^{m+1-k}\right)-a_{m+1-k, k-1}^{m+1-k} .
\end{aligned}
$$

This shows that (2.4) is verified. On letting

$$
c_{m+1-k, k-}^{0}=\left(\chi_{m+1-k, k}^{1} \circ \cdots \circ \chi_{m+1-k, k}^{m+1-k}\right)\left(c_{m+1-k, k}^{m+1-k}\right),
$$

$k=0, \ldots, m+1$, as well as

$$
\begin{gathered}
c^{0}=\sum_{k=0}^{m+1} z_{m+1-k, k}^{0}\left(c_{m+1-k, k}^{0}\right) \\
a^{0}=\sum_{k=0}^{m} z_{m-k, k}^{0}\left(a_{m-k, k}^{0}\right)
\end{gathered}
$$

we infer from (2.4) that $\Delta_{m+1}^{0}\left(c^{0}\right)=a^{0}$. Setting $\chi=\chi^{1} \circ \cdots \circ \chi^{m+1}$ we see that

$$
H_{m}(\bar{\chi})([a])=[\bar{\chi}(a)]=0 .
$$

We observe that both $\alpha_{m}^{j}: H_{m}\left(A_{*, *}^{j}\right) \rightarrow H_{m}\left(L_{*}\right), j=0, m+1$, are isomorphisms according to hypothesis (3) and Proposition 2.1. Lemma 2.2 in turn implies that $H_{m}(\bar{\chi}): H_{m}\left(A_{*, *}^{m+1}\right) \rightarrow H_{m}\left(A_{*, *}^{0}\right)$ is an isomorphism as well, and equality (2.5) implies that $[a]=0$, showing that $\beta_{m}^{m+1}$ is injective.

For the Definition of inverse limit we refer to Appendix A. 
Theorem 2.4. Assume that:

(1) $(J, \preceq)$ is a (nonempty) directed set;

(2) $m \geq 1$ is an integer;

(3) $\left(\left(A_{*, *}^{j} ; \Phi_{*, *}^{j} ; \Psi_{*, *}^{j}\right),\left(L_{*} ; \Psi_{*}\right),\left(M_{*}^{j} ; \Phi_{*}^{j}\right)\right), j \in J$, are augmented double chain complexes (observe that $\left(L_{*} ; \Psi_{*}\right)$ doesn't depend upon $\left.j\right)$;

(4) for every $j \in J$ and every $q=0, \ldots, m+1$, the following sequence is exact:

$$
A_{m+1-q, q}^{j} \stackrel{\Phi_{m+1-q, q}^{j}}{\longrightarrow} \cdots \stackrel{\Phi_{2, q}^{j}}{\longrightarrow} A_{1, q}^{j} \stackrel{\Phi_{1, q}^{j}}{\longrightarrow} A_{0, q}^{j} \stackrel{\Phi_{0, q}^{j}}{\longrightarrow} L_{q} \longrightarrow 0
$$

(5) for every $j \in J$ and $p=0, \ldots, m+1$, the morphism $\Psi_{p, 0}^{j}$ is surjective;

(6) for every $j_{1}, j_{2} \in J$ such that $j_{1} \preceq j_{2}$ there is a collection $\chi^{j_{1}, j_{2}}$ of morphisms of double chain complexes

$$
\left(A_{*, *}^{j_{2}} ; L_{*} ; M_{*}^{j_{2}}\right) \rightarrow\left(A_{*, *}^{j_{1}} ; L_{*} ; M_{*}^{j_{1}}\right)
$$

meeting the following requirements:

(6.1) for every $j \in J$ the identity belongs to $\chi^{j, j}$;

(6.2) for every $j_{1}, j_{2} \in J$ with $j_{1} \preceq j_{2}$ and every $\chi^{j_{1}, j_{2}}, \tilde{\chi}^{j_{1}, j_{2}} \in \chi^{j_{1}, j_{2}}$ one has $H_{m}\left(\chi_{*,-1}^{j_{1}, j_{2}}\right)=H_{m}\left(\tilde{\chi}_{*,-1}^{j_{1}, j_{2}}\right)$;

(6.3) for every $j_{1}, j_{2}, j_{3} \in J$ with $j_{1} \preceq j_{2} \preceq j_{3}$ and every $\chi^{j_{1} j_{2}} \in \chi^{j_{1}, j_{2}}$, $\chi^{j_{2}, j_{3}} \in \chi^{j_{2}, j_{3}}$ one has $\chi^{j_{1}, j_{2}} \circ \chi^{j_{2}, j_{3}} \in \chi^{j_{1}, j_{3}} ;$

(6.4) for every $j_{1} \in J$ there exists $j_{2} \in J$ with $j_{1} \preceq j_{2}$ and there exists $\chi^{j_{1}, j_{2}} \in \chi^{j_{1}, j_{2}}$ such that

$$
\chi^{j_{1}, j_{2}}\left(\operatorname{ker} \Psi_{p, q}^{j_{2}}\right) \subset \operatorname{im} \Psi_{p, q+1}^{j_{1}}
$$

whenever $p, q=0,1,2, \ldots$

Then there is an ismorphism

$$
\Gamma_{m}: H_{m}\left(L_{*}\right) \rightarrow \lim _{j \in J} H_{m}\left(M_{*}^{j}\right),
$$

where the inverse limit is taken over the homomorphisms

$$
H_{m}\left(\chi_{*,-1}^{j_{1}, j_{2}}\right): H_{m}\left(M_{*}^{j_{2}}\right) \rightarrow H_{m}\left(M_{*}^{j_{1}}\right) .
$$

Furthermore $\Gamma_{m}$ is characterized by the following property: for every $j \in J$ one has

$$
p_{j} \circ \Gamma_{m}=\beta_{m}^{j} \circ\left(\alpha_{m}^{j}\right)^{-1}
$$

where $p_{j}: \lim _{i \in J} H_{m}\left(M_{*}^{i}\right) \rightarrow H_{m}\left(M_{*}^{j}\right)$ is the canonical projection. 
Proof. Apply inductively hypothesis (6.4) to infer the existence of $i_{0} \preceq i_{1} \preceq$ $\ldots \preceq i_{m+1}$, elements of $J$, and of morphisms $\chi^{i_{k}, i_{k+1}} \in \chi^{i_{k}, i_{k+1}}, k=0, \ldots, m$, such that

$$
\chi^{i_{k}, i_{k+1}}\left(\operatorname{ker} \Psi_{p, q}^{i_{k+1}}\right) \subset \operatorname{im} \Psi_{p, q+1}^{i_{k}},
$$

$k=0, \ldots, m, p, q=0,1,2, \ldots$. Next we let $\hat{J}=J \cap\left\{j: i_{m+1} \preceq j\right\}$ and we will define an isomorphism

$$
\gamma_{m}: H_{m}\left(A_{*, *}^{i_{m+1}}\right) \rightarrow \lim _{j \in \hat{J}} H_{m}\left(M_{*}^{j}\right)
$$

Recalling that $\left(\alpha_{m}^{i_{m+1}}\right)^{-1}: H_{m}\left(L_{*}\right) \rightarrow H_{m}\left(A_{*, *}^{i_{m+1}}\right)$ is an isomorphism (as follows from Proposition 2.1) the existence of $\Gamma_{m}$ will readily result from the fact that $\hat{J}$ is cofinal in $J$.

Given $[a] \in H_{m}\left(A_{*, *}^{i_{m+1}}\right)$ we let

$$
\gamma_{m}([a])=\left(\left(\beta_{m}^{j} \circ H_{m}\left(\overline{\chi^{i_{m+1}, j}}\right)^{-1}\right)([a])\right)_{j \in \hat{J}}
$$

(recall that $H_{m}\left(\overline{\chi^{i_{m+1}, j}}\right)$ is an isomorphism according to Proposition 2.1 and Lemma 2.2). It is trivial to check that

$$
\gamma_{m}([a]) \in \lim _{\leftarrow \in \hat{J}} H_{m}\left(M_{*}^{j}\right),
$$

so that $\gamma_{m}$ is a well defined homomorphism.

Assume that $\gamma_{m}([a])=0$. Then also $\beta_{m}^{i_{m+1}}([a])=0$, whence $[a]=0$ since $\beta_{m}^{i_{m+1}}$ is injective according to Theorem 2.3(7). This shows that $\gamma_{m}$ is onte-to-one.

We now turn to showing that $\gamma_{m}$ is surjective. Let

$$
\left(\left[b^{j}\right]\right)_{j \in \hat{J}} \in \lim _{j \in \hat{J}} H_{m}\left(M_{*}^{j}\right)
$$

and fix some $j_{0} \in \hat{J}$. Applying inductively hypothesis (6.4) we find $j_{1}, \ldots, j_{m}$ $\in \hat{J}$ such that $j_{0} \preceq j_{1} \preceq \ldots \preceq j_{m}$ and

$$
\chi^{j_{k}, j_{k+1}}\left(\operatorname{ker} \Psi_{p, q}^{j_{k+1}}\right) \subset \operatorname{im} \Psi_{p, q+1}^{j_{k}},
$$

$k=1, \ldots, m, p, q=0,1,2, \ldots$ According to Theorem 2.3(6) there exists $\left[a^{j_{0}}\right] \in H_{m}\left(A_{*, *}^{j_{0}}\right)$ such that

$$
\beta_{m}^{j_{0}}\left(\left[a^{j_{0}}\right]\right)=H_{m}\left(\chi_{*,-1}^{j_{0}, j_{1}} \circ \cdots \circ \chi_{*,-1}^{j_{m-1}, j_{m}}\right)\left(\left[b^{j_{m}}\right]\right)=\left[b^{j_{0}}\right] .
$$

On letting $\left[\hat{a}^{j_{0}}\right]=H_{m}\left(\overline{\chi^{i_{m+1}, j_{0}}}\right)\left(\left[a^{j_{0}}\right]\right) \in H_{m}\left(A_{*, *}^{i_{m+1}}\right)$ we see that

$$
\beta_{m}^{i_{m+1}}\left(\left[\hat{a}^{j_{0}}\right]\right)=\left[b^{i_{m+1}}\right] .
$$


It now follows from $(2.8)$ and the injectivity of $\beta_{m}^{i_{m+1}}$ that $\left[\hat{a}^{j_{0}}\right]=\left[\hat{a}^{j_{0}^{\prime}}\right]$ whenever $j_{0}, j_{0}^{\prime} \in \hat{J}$. Let $[\hat{a}]$ denote the common value. Then it follows from the definition of $\left[\hat{a}^{j}\right], j \in \hat{J}$, together with (2.7), that

$$
\gamma_{m}([\hat{a}])=\left(\left[b^{j}\right]\right)_{j \in \hat{J}} \cdot
$$

It remains to show that $\Gamma_{m}$ is characterized by the relations $p_{j} \circ \Gamma_{m}=$ $\beta_{m}^{j} \circ\left(\alpha_{m}^{j}\right)^{-1}, j \in J$. First notice that there is clearly only one homomorphism $\Gamma_{m}$ verifying these equations. Next observe that the definition of $\gamma_{m}$ implies that whenever $i_{m+1} \preceq j$ one has

$$
\begin{aligned}
p_{j} \circ \Gamma_{m} & =\beta_{m}^{j} \circ H_{m}\left(\overline{\chi^{i_{m+1}, j}}\right)^{-1} \circ\left(\alpha_{m}^{i_{m+1}}\right)^{-1} \\
& =\beta_{m}^{j} \circ\left(\alpha_{m}^{i_{m+1}} \circ H_{m}\left(\overline{\chi^{i_{m+1}, j}}\right)\right)^{-1} \\
& =\beta_{m}^{j} \circ\left(H_{m}\left(\chi_{-1, *}^{i_{m+1}, j}\right) \circ \alpha_{m}^{j}\right)^{-1} \\
& =\beta_{m}^{j} \circ\left(\alpha_{m}^{j}\right)^{-1}
\end{aligned}
$$

because $H_{m}\left(\chi_{-1, *}^{i_{m+1}, j}\right)=\operatorname{id}_{H_{m}\left(L_{*}\right)}$. Since the required equation holds for all $j \in J$ with $i_{m+1} \preceq j$, it clearly holds for all $j \in J$.

Theorem 2.5. Let $m \geq 1$ be an integer and let $J$ and $K$ be (nonempty) directed sets. Consider two families of augmented double chain complexes $\left(\left(A_{*, *}^{j} ; \Phi_{*, *}^{j} ; \Psi_{*, *}^{j}\right),\left(L_{*}, \Psi_{*}\right),\left(M_{*}^{j}, \Phi_{*}^{j}\right)\right), j \in J$, and $\left(\left(\tilde{A}_{*, *}^{k} ; \tilde{\Phi}_{*, *}^{k} ; \tilde{\Psi}_{*, *}^{k}\right),\left(\tilde{L}_{*} ; \tilde{\Psi}_{*}\right)\right.$, $\left.\left(\tilde{M}_{*}^{k} ; \tilde{\Phi}^{k}\right)\right), k \in K$, verifying hypotheses (4), (5) and (6) of Theorem 2.4. Assume that $\varphi: K \rightarrow J$ is an order preserving function, and assume that for each $k \in K$ there is a morphism of augmented double chain complexes

$$
f^{k}:\left(A_{*, *}^{\varphi(k)} ; L_{*} ; M_{*}^{\varphi(k)}\right) \rightarrow\left(\tilde{A}_{*, *}^{k} ; \tilde{L}_{*} ; \tilde{M}_{*}^{k}\right)
$$

verifying the following commutativity condition:

$$
H_{m}\left(\overline{f^{k_{1}}}\right) \circ H_{m}\left(\overline{\chi^{\varphi\left(k_{1}\right), \varphi\left(k_{2}\right)}}\right)=H_{m}\left(\overline{\tilde{\chi}^{k_{1}, k_{2}}}\right) \circ H_{m}\left(\overline{f^{k_{2}}}\right)
$$

whenever $k_{1}, k_{2} \in K$ and $k_{1} \preceq k_{2}$. We also assume that the homomorphism $H_{m}\left(f_{-1, *}^{k}\right): H_{m}\left(L_{*}\right) \rightarrow H_{m}\left(\tilde{\tilde{L}}^{*}\right)$ doesn't depend upon its index $k \in K$ and is simply denoted $H_{m}\left(f_{-1, *}\right)$ hereunder. Then the following diagram commutes:

$$
\begin{aligned}
& H_{m}\left(L_{*}\right) \stackrel{\Gamma_{m}}{\longrightarrow} \lim _{\leftarrow \in \hat{J}} H_{m}\left(M_{*}^{j}\right) \\
& H_{m}\left(f_{-1, *}\right) \downarrow \stackrel{\lim }{\leftarrow} H_{m}\left(f_{*,-1}^{k}\right) \downarrow \\
& H_{m}\left(\tilde{L}_{*}\right) \stackrel{\tilde{\Gamma}_{m}}{\longrightarrow} \lim _{k \in \hat{K}} H_{m}\left(\tilde{M}_{*}^{k}\right)
\end{aligned}
$$


Proof. We use the characterization of $\Gamma_{m}$ and $\tilde{\Gamma}_{m}$ given in Theorem 2.4 to infer that for each $k \in K$ :

$$
\begin{aligned}
\tilde{p}_{k} \circ \lim _{\leftarrow} H_{m}\left(f_{*,-1}^{k}\right) & \circ \Gamma_{m}=H_{m}\left(f^{k}\right) \circ p_{\varphi(k)} \circ \Gamma_{m}=H_{m}\left(f^{k}\right) \circ \beta_{m}^{\varphi(k)} \circ\left(\alpha_{m}^{\varphi(k)}\right)^{-1} \\
& =\tilde{\beta}_{m}^{k} \circ H_{m}\left(\overline{f^{k}}\right) \circ\left(\alpha_{m}^{\varphi(k)}\right)^{-1}=\tilde{\beta}_{m}^{k} \circ\left(\tilde{\alpha}_{m}^{k}\right)^{-1} \circ H_{m}\left(f_{-1, *}\right) \\
& =\tilde{p}_{k} \circ \tilde{\Gamma}_{m} \circ H_{m}\left(f_{-1, *}\right) .
\end{aligned}
$$

This completes the proof.

\section{2. Čech versus singular homology}

Our reference for simplicial, singular and Čech homology is [10]. We assume the reader is familiar with simplicial and singular theories, and we start by quickly reviewing Čech homology.

Let $X$ be a topological space and let $\mathcal{O}(X)$ denote the collection of open subsets of $X$. An open cover of $X$ (subsequently abbreviated as cover) consists in a nonempty set $\mathcal{U}$ and a map $a: \mathcal{U} \rightarrow \mathcal{O}(X)$ such that $X=$ $\cup\{a(U): U \in \mathcal{U}\}$. A cover $(\mathcal{V}, b)$ is a refinement of $(\mathcal{U}, a)$ if for every $V \in \mathcal{V}$ there exists $U \in \mathcal{U}$ such that $b(V) \subset a(U)$ : we will abbreviate this by the notation $\mathcal{U} \preceq \mathcal{V}$. In fact we will from now on identify a cover $(\mathcal{U}, a)$ with its domain $\mathcal{U}$, and the open sets $a(U)$ with their index $U$. A cover of $X$ differs from a subset $\mathcal{O}^{\prime} \subset \mathcal{O}(X)$ with $X=\cup \mathcal{O}^{\prime}$ merely in that the same open set $U \subset X$ may appear several distinct times in a cover. Sometimes we will choose an other index set $I$ for a cover $\mathcal{U}$ and write $\mathcal{U}=\left\{U_{i}: i \in I\right\}$. The relation $\preceq$ on the collection $\operatorname{Cov}(X)$ of covers of $X$ makes it into a directed set. With each $\mathcal{U} \in \operatorname{Cov}(X)$ we associate its nerve, denoted $N(\mathcal{U})$, which is an (abstract) simplicial complex defined as follows: its vertices are the elements of $\mathcal{U}$, and for each $k=1,2, \ldots$, a subset $\mathcal{S} \subset \mathcal{U}$ with card $\mathcal{S}=k+1$ belongs to the $k$ skeleton of $N(\mathcal{U})$ if and only if $\cap \mathcal{S} \neq \emptyset$. Given an abelian group $G$ (or an $R$-module), the simplicial homology groups of $N(\mathcal{U})$ with coefficients in $G$ will be denoted $H_{q}(N(\mathcal{U}) ; G), q=0,1,2, \ldots$. Now if $\mathcal{U}, \mathcal{V} \in \operatorname{Cov}(X)$ and $\mathcal{U} \preceq \mathcal{V}$ then there exists at least one map $\iota: \mathcal{V} \rightarrow \mathcal{U}$ such that $V \subset \iota(V)$ whenever $V \in \mathcal{V}$. Each such map is called a refinement projection and induces the same homomorphism in homology:

$$
\pi_{q}^{\mathcal{U}, \mathcal{V}}: H_{q}(N(\mathcal{V}) ; G) \rightarrow H_{q}(N(\mathcal{U}) ; G)
$$

Therefore these groups and homomorphisms form an inverse system in one of the categories $\mathrm{Ab}, \mathrm{Mod}_{R}$, and we define the Čech homology groups of $X$ as follows:

$$
\check{H}_{q}(X ; G)=\lim _{\mathfrak{U} \in \operatorname{Cov}(X)} H_{q}(N(\mathcal{U}) ; G)
$$


Next, if $X$ and $Y$ are topological spaces and $f: X \rightarrow Y$ is continuous then there is a morphism of inverse systems

$$
\left(H_{q}(N(\mathcal{U}) ; G), \pi_{q}\right) u \in \operatorname{Cov}(X) \rightarrow\left(H_{q}(N(\mathcal{V}) ; G), \pi_{q}\right) v_{\mathcal{C}} \operatorname{Cov}(Y)
$$

defined as follows. We let $\varphi: \operatorname{Cov}(Y) \rightarrow \operatorname{Cov}(X)$ be such that $\varphi(\mathcal{V})=$ $\left\{f^{-1}(V): V \in \mathcal{V}\right\}, \mathcal{V} \in \operatorname{Cov}(Y)$. For each $\mathcal{V} \in \operatorname{Cov}(Y)$ we let

$$
H_{q}\left(f_{\mathcal{V}}\right): H_{q}(N(\varphi(\mathcal{V})) ; G) \rightarrow H_{q}(N(\mathcal{V}) ; G)
$$

be induced by the map $f_{\mathcal{V}}: N(\varphi(\mathcal{V})) \rightarrow N(\mathcal{V})$ which to $f^{-1}(V) \in \varphi(\mathcal{V})$ associates $V \in \mathcal{V}$. The limiting morphism is denoted

$$
\check{H}_{q}(f): \check{H}_{q}(X ; G) \rightarrow \check{H}_{q}(Y ; G)
$$

The relative Čech homology groups of a pair $(X, A)$ are defined in analogous way: the inverse limit is now taken over the directed set $\operatorname{Cov}(X, A)$ of pairs $\left(\mathcal{U}_{X}, \mathcal{U}_{A}\right)$ where $\mathcal{U}_{X}$ (respectively $\mathcal{U}_{A}$ ) is a of $X$ (respectively of $A$ ) and $\mathcal{U}_{A} \subset \mathcal{U}_{X}$.

Remark 2.6. Let $\mathrm{A}$ denote the category of arbitrary pairs of topological spaces and their continuous maps. The functors $\check{H}_{q}: \mathrm{A} \rightarrow \mathrm{Ab}$ and $\check{H}_{q}: \mathrm{A} \rightarrow$ $\operatorname{Mod}_{R}$ defined above (according to whether $G$ is an object of $A b$ or $\operatorname{Mod}_{R}$ ) verify the axioms of Eilenberg-Steenrod except for the Exactness Axiom. This can be traced to the fact that the inverse limit of an exact sequence of abelian groups or $R$ modules doesn't need to be exact (see [10, pp 225-226]). However the Exactness Axiom is verified if we restrict ourselves to consider-

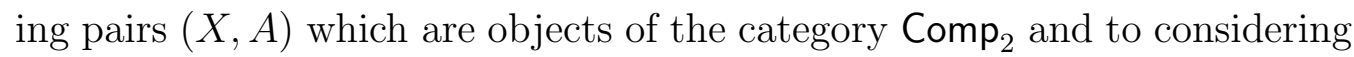
coefficients groups $G$ which are objects of the category $\mathrm{Ab}_{\mathrm{c}}$. In that case the directed subset $\operatorname{Cov}^{f}(X, A)$ consisting of finite covers is cofinal in $\operatorname{Cov}(X, A)$ ([10, Chapter IX, Lemma 3.4]). Clearly, if $\left(\mathcal{U}_{X}, \mathcal{U}_{A}\right) \in \operatorname{Cov}^{f}(X, A)$ then the simplicial homology groups $H_{q}\left(N\left(\mathcal{U}_{X}\right), N\left(\mathcal{U}_{A}\right) ; G\right)$ belong to $\mathrm{Ab}_{\mathrm{c}}$ and this category has inverse limits, so that $\check{H}_{q}: \mathrm{Comp}_{2} \rightarrow \mathrm{Ab}_{\mathrm{c}}$. The assertion now follows from the fact that the inverse limit of an exact sequence in $A b_{c}$ is still exact (see [10, Chapter VIII, Theorem 5.6]). The need for the Exactness Axiom in validating "cut and paste" arguments is the reason why E.R. Reifenberg considered only coefficient groups belonging to $A b_{c}$ in his treatment of the Plateau problem in [16] — see [6] for further developments.

We now state the "continuity" property of Čech homology which is essential in proving the existence Theorems of [6], see [10, Chapter X, Theorem 3.1] for a proof. 
Theorem 2.7. Let $q=0,1,2, \ldots$ and let $G$ be an object of $\operatorname{Ab}_{c}$ or $\operatorname{Mod}_{R}$. Then the functors

$$
\operatorname{Inv}\left(\operatorname{Comp}_{2}\right) \rightarrow \mathrm{Ab}_{\mathrm{c}}\left(\text { resp. } \operatorname{Mod}_{R}\right):(\mathbf{X}, \mathbf{A}) \mapsto \lim _{\leftarrow} \check{H}_{q}(\mathbf{X}, \mathbf{A} ; G)
$$

and

$$
\operatorname{lnv}\left(\operatorname{Comp}_{2}\right) \rightarrow \mathrm{Ab}_{\mathrm{c}}\left(\text { resp. } \operatorname{Mod}_{R}\right):(\mathbf{X}, \mathbf{A}) \mapsto \check{H}_{q}(\lim (\mathbf{X}, \mathbf{A}) ; G)
$$

are naturally equivalent.

The remaining part of this section is devoted to comparing the singular homology groups $H_{q}(X, A ; G)$ and the Čech homology groups $\check{H}_{q}(X, A ; G)$ under certain "local connectedness" assumptions on the pair $(X, A)$. The following is taken from [5, Theorem 4.4].

Proposition 2.8. Assume that

(1) $X$ is a paracompact topological space;

(2) $\mathrm{C}$ is one of the categories $\mathrm{Ab}, \mathrm{Ab}_{\mathrm{c}}, \operatorname{Mod}_{R}$ and $H: \mathrm{O}(X) \rightarrow \mathrm{C}$ is a functor with the following property: for each $x \in X$ and each open neighborhood $U$ of $x$, there is an open neighborhood $U^{\prime} \subset U$ of $x$ such that $H\left(U^{\prime}\right) \rightarrow H(U)$ is trivial;

(3) $\mathcal{U}$ is a cover of $X$.

Then there exists a $\mathcal{W} \succeq \mathcal{U}$ of $X$ and a refinement projection $u: \mathcal{W} \rightarrow \mathcal{U}$ such that if $W_{0}, \ldots, W_{p} \in \mathcal{W}$ and $W_{0} \cap \ldots \cap W_{p} \neq \emptyset$ then the homomorphism

$$
H\left(W_{0} \cap \ldots \cap W_{p}\right) \rightarrow H\left(u\left(W_{0}\right) \cap \ldots \cap u\left(W_{p}\right)\right)
$$

is trivial.

Proof. Since $X$ is paracompact there exists a locally finite $\mathcal{U}_{0} \succeq \mathcal{U}$ of $X$ and, for each $U \in \mathcal{U}_{0}$, an open set $o(U)$ such that $\operatorname{Clos} o(U) \subset U$, and also $X=\cup\left\{o(U): U \in \mathcal{U}_{0}\right\}$. Whenever $V \subset X$ is open and $x \in X$ we let $\mathcal{U}_{0}(V)=\mathcal{U}_{0} \cap\{U: U \cap V \neq \emptyset\}$ and $\mathcal{U}_{0}(x)=\mathcal{U}_{0} \cap\{U: x \in U\}$. Now fix $U \in \mathcal{U}_{0}$ and $x \in o(U)$. Since $\mathcal{U}_{0}$ is locally finite there exists an open neighborhood of $x$, say $U(x)$, such that card $\mathcal{U}_{0}(U(x))<\infty$. Therefore $U^{\prime}(x)=U(x) \cap\left(\cap \mathcal{U}_{0}(x)\right)$ is an open neighborhood of $x$. We also observe that

$$
F(x)=\cup\left\{\operatorname{Clos} o(U): U \in \mathcal{U}_{0}(U(x)) \sim \mathcal{U}_{0}(x)\right\}
$$

is a closed set not containing $x$. Whence $x$ has an open neighborhood $U^{\prime \prime}(x) \subset U^{\prime}(x)$ such that $U^{\prime \prime}(x) \cap F(x)=\emptyset$. Finally $x$ admits an even smaller open neighborhood, denoted $W(x, U) \subset U^{\prime \prime}(x)$ such that

$$
H(W(x, U)) \rightarrow H\left(U^{\prime \prime}(x)\right)
$$

is trivial. 
We now claim that the following holds. For every $U_{0}, \ldots, U_{p} \in \mathcal{U}_{0}$, if $W(x, U) \cap o\left(U_{0}\right) \cap \ldots \cap o\left(U_{p}\right) \neq \emptyset$ then $W(x, U) \subset U_{0} \cap \ldots \cap U_{p}$ and

$$
H(W(x, U)) \rightarrow H\left(U_{0} \cap \ldots \cap U_{p}\right)
$$

is trivial. Indeed, for each $i=0, \ldots, p$ we must have $U_{i} \in \mathcal{U}_{0}(x)$ by construction. Therefore $W(x, U) \subset U^{\prime \prime}(x) \subset U^{\prime}(x) \subset U_{i}$. This proves the first assertion whereas the second follows from the factorization

$$
H(W(x, U)) \rightarrow H\left(U^{\prime \prime}(x)\right) \rightarrow H\left(U_{0} \cap \ldots \cap U_{p}\right) .
$$

We define $\mathcal{W}=\cup\left\{W(x, U): U \in \mathcal{U}_{0}\right.$ and $\left.x \in o(U)\right\}$ which clearly covers $X$, and a refinement projection $u: \mathcal{W} \rightarrow \mathcal{U}$ such that if $W \in \mathcal{W}$ then $W=W(x, u(W))$ for some $x \in X$. If $W_{i}=W\left(x_{i}, U_{i}\right) \in \mathcal{W}, U_{i}=u\left(W_{i}\right)$, $i=0, \ldots, p$, and $W_{0} \cap \ldots \cap W_{p} \neq \emptyset$ then the relation

$$
W\left(x_{0}, U_{0}\right) \cap \ldots \cap W\left(x_{p}, U_{p}\right) \subset o\left(U_{0}\right) \cap \ldots \cap o\left(U_{p}\right)
$$

implies that

$$
W\left(x_{0}, U_{0}\right) \subset o\left(U_{0}\right) \cap \ldots \cap o\left(U_{p}\right)
$$

and, in turn, according to the previous paragraph, that $W\left(x_{0}, U_{0}\right) \subset U_{0} \cap$ $\ldots \cap U_{p}$ and that

$$
H\left(W_{0} \cap \ldots \cap W_{p}\right) \rightarrow H\left(W\left(x_{0}, U_{0}\right)\right) \rightarrow H\left(U_{0} \cap \ldots \cap U_{p}\right)
$$

is trivial.

We now introduce some notations preliminary to the definition of a cosheaf. Let $X$ be a topological space; let $\mathrm{C}$ be one of the categories $\mathrm{Ab}$, $\mathrm{Ab}_{\mathrm{c}}$ and $\operatorname{Mod}_{R}$; let $F: \mathrm{O}(X) \rightarrow \mathrm{C}$ be a functor; whenever $U \subset V \subset X$ are open let $\varphi_{V ; U}$ denote the unique morphism in $\mathrm{O}(X)$ from $U$ to $V$; and let $\mathcal{U}=\left\{U_{i}: i \in I\right\}$ be a countable family of open subsets $X$. Whenever $i_{0}, \ldots, i_{p} \in I, p \geq 0$, we abbreviate

$$
U_{i_{0}, \ldots, i_{p}}=\cap_{k=0}^{p} U_{i_{k}} .
$$

From now on we will always assume that some choice of an order $\leq$ on $I$ has been made. For each $p \geq 1$ we define a homomorphism

$$
\Phi_{p, F}^{\mathcal{U}}: \bigoplus_{i_{0}<\ldots<i_{p}} F\left(U_{i_{0}, \ldots, i_{p}}\right) \rightarrow \bigoplus_{i_{0}<\ldots<i_{p-1}} F\left(U_{i_{0}, \ldots, i_{p-1}}\right)
$$

as follows: given $i_{0}<\ldots<i_{p}$ and $a \in F\left(U_{i_{0}, \ldots, i_{p}}\right)$ we set

$$
\Phi_{p, F}^{\mathcal{u}}(a)=\sum_{k=0}^{p}(-1)^{k} F\left(\varphi_{U_{i_{0}, \ldots, \widehat{k_{k}}, \ldots, i_{p}} ; U_{i_{0}, \ldots, i_{p}}}\right)(a) .
$$


Of course, if card $\mathcal{U}=\nu$ then $\Phi_{p, F}^{\mathcal{U}}=0$ whenever $p \geq \nu$. We also define

$$
\Phi_{0, F}^{\mathcal{U}}: \bigoplus_{i \in I} F\left(U_{i}\right) \rightarrow F(\cup \mathcal{U})
$$

by

$$
\Phi_{0, F}^{\mathcal{u}}\left(\sum a_{i}\right)=\sum F\left(\varphi \cup u ; U_{i}\right)\left(a_{i}\right) .
$$

One readily checks that $\Phi_{p+1, F}^{\mathcal{U}} \circ \Phi_{p, F}^{\mathcal{U}}=0, p \geq 0$.

Definition 2.9. A cosheaf on a topological space $X$ is a functor $F: \mathrm{O}(X) \rightarrow \mathrm{C}$ (where $C$ is one of the categories $A b, A b_{c}$ and $\operatorname{Mod}_{R}$ ) verifying the following condition. For every countable family $\mathcal{U}=\left\{U_{i}: i \in I\right\}$ of open subsets of $X$, the following sequence is exact:

$$
\begin{aligned}
\ldots \stackrel{\Phi_{p+1, F}^{u}}{\longrightarrow} \bigoplus_{i_{0}<\ldots<i_{p}} F\left(U_{i_{0}, \ldots, i_{p}}\right) \stackrel{\Phi_{p, F}^{u}}{\longrightarrow} & \cdots \stackrel{\Phi_{2, F}^{u}}{\longrightarrow} \bigoplus_{i_{0}<i_{1}} F\left(U_{i_{0}, i_{1}}\right) \\
& \stackrel{\Phi_{1, F}^{u}}{\longrightarrow} \bigoplus_{i \in I} F\left(U_{i}\right) \stackrel{\Phi_{0, F}^{u}}{\longrightarrow} F(\cup \mathcal{U}) \longrightarrow 0
\end{aligned}
$$

Proposition 2.10. Let $X$ be a topological space, let $\mathrm{C}$ be one of the categories $\mathrm{Ab}, \mathrm{Ab}_{\mathrm{c}}$ and $\mathrm{Mod}_{R}$, and let $F: \mathrm{O}(X) \rightarrow \mathrm{C}$ be a functor. Assume that

(1) for every pair of open sets $U_{0}, U_{1} \subset X$ the following sequence is exact:

$$
F\left(U_{0} \cap U_{1}\right) \stackrel{\Phi_{1, F}^{u}}{\longrightarrow} F\left(U_{0}\right) \oplus F\left(U_{1}\right) \stackrel{\Phi_{0, F}^{u}}{\longrightarrow} F\left(U_{0} \cup U_{1}\right) \longrightarrow 0
$$

where $\mathcal{U}=\left\{U_{0}, U_{1}\right\}$

(2) for every countable family of open sets $\mathcal{U}=\left\{U_{i}: i \in I\right\}$ which is directed upwards by inclusion, the natural homomorphism

$$
\lim _{i \in I} F\left(U_{i}\right) \longrightarrow F(\cup \mathcal{U})
$$

is an isomorphism.

Then $F$ is a cosheaf.

Proof. Given a family of open sets $\mathcal{U}=\left\{U_{i}: i \in I\right\}$ we need to check that the sequence (2.9) of Definition 2.9 is exact. Exactness at $F(\cup \mathcal{U})$ follows from hypothesis (2) together with the exactness at $F\left(\cup \mathcal{U}^{\prime}\right)$ of the sequence associated to $\mathcal{U}^{\prime}$ for every finite family $\mathcal{U}^{\prime}$. Similarly, exactness at $\oplus_{i_{0}<\ldots<i_{p}} F\left(U_{i_{0}, \ldots, i_{p}}\right)$ of the sequence associated to $\mathcal{U}$ follows from the exactness at the same node of the sequence associated to a finite family $\mathcal{U}^{\prime}$. 
Henceforth we will assume $\mathcal{U}=\left\{U_{0}, \ldots, U_{p}\right\}$ is finite and we will prove that the sequence (2.9) is exact by induction on $p$; the case $p=1$ corresponds to hypothesis (1). Suppose the conclusion holds for $p$ and let $\mathcal{U}=\left\{U_{0}, \ldots, U_{p+1}\right\}$ be a family of $p+1$ open subsets of $X$. We put $\mathcal{V}=\left\{U_{0}, \ldots, U_{p}\right\}, V=\cup \mathcal{V}, \mathcal{U}^{\prime}=\left\{U_{0} \cap U_{p+1}, \ldots, U_{p} \cap U_{p+1}\right\}, \mathcal{W}=\left\{V, U_{p+1}\right\}$, and we consider the following diagram:

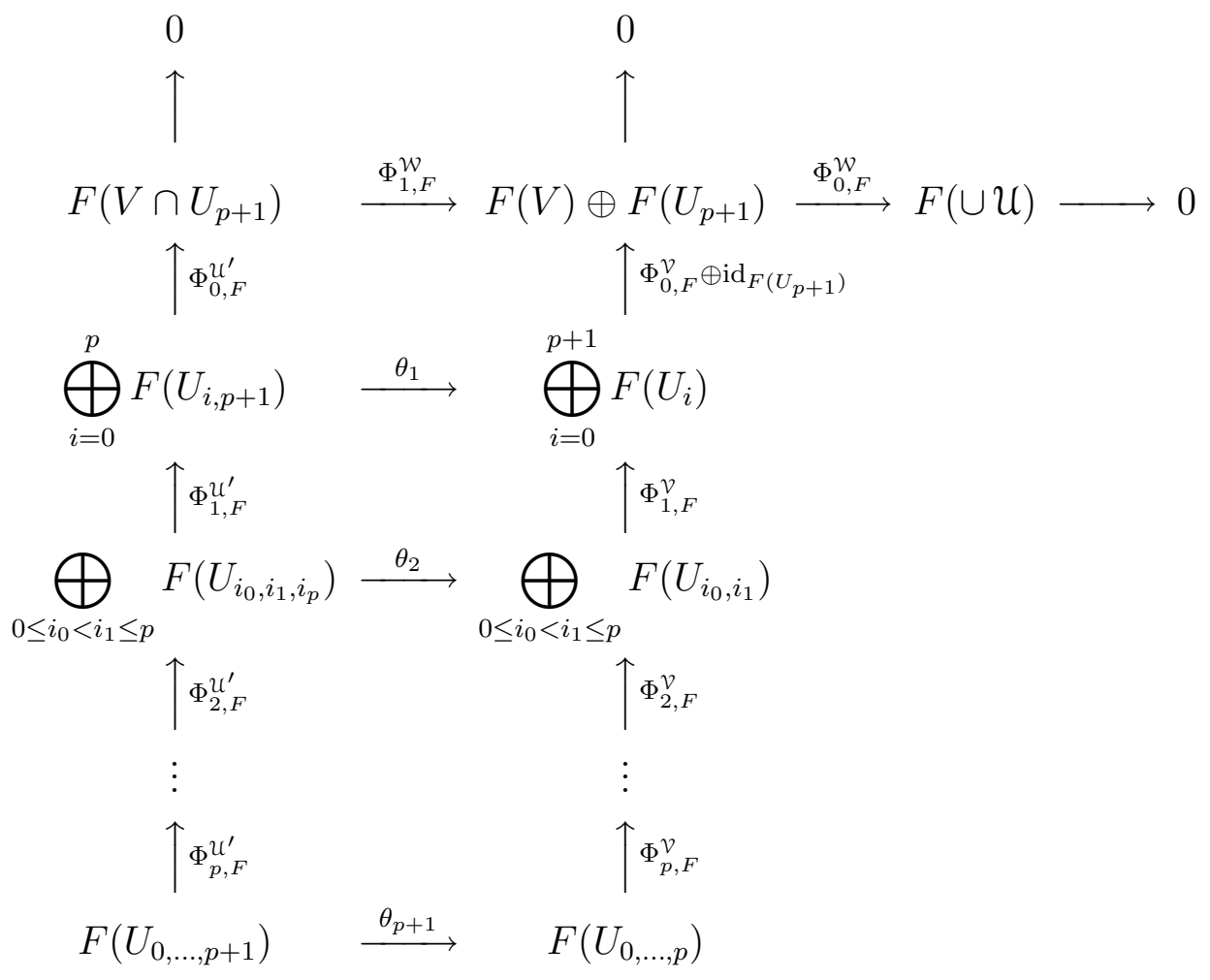

The first row is exact according to hypothesis (1) applied to the pair $V$, $U_{p+1}$. The columns are exact by the induction hypothesis applied respectively to $\mathcal{U}^{\prime}$ (left column) and to $\mathcal{V}$ (right column). The homomorphisms $\theta_{j}, j=1, \ldots, p+1$, are defined in order to make the diagram commute: if $a \in F\left(U_{i, p+1}\right), i=0, \ldots, p$, then $\theta_{1}(a)=-F\left(\varphi_{U_{i} ; U_{i, p+1}}\right)+F\left(\varphi_{U_{p+1} ; U_{i, p+1}}\right)$; if $j=2, \ldots, p+1,0 \leq i_{1}<\ldots<i_{j} \leq p, a \in F\left(U_{i_{1}, \ldots, i_{j}, i_{p+1}}\right)$ then $\theta_{j}(a)=-F\left(\varphi_{U_{i_{1}, \ldots, i_{j}} ; U_{i_{1}, \ldots, i_{j}, i_{p+1}}}\right)$. We want to show that the following sequence is exact:

$$
\begin{aligned}
F\left(U_{0, \ldots, p+1}\right) \stackrel{\Phi_{p+1, F}^{u}}{\longrightarrow} \cdots \stackrel{\Phi U_{2, F}}{\longrightarrow} \bigoplus_{0 \leq i_{0}<i_{1} \leq p} F\left(U_{i_{0}, i_{1}}\right) \\
\\
\stackrel{\Phi_{1, F}^{\mathcal{u}}}{\longrightarrow} \bigoplus_{i=0}^{p} F\left(U_{i}\right) \stackrel{\Phi_{0, F}^{u}}{\longrightarrow} F(\cup \mathcal{U}) \longrightarrow 0 .
\end{aligned}
$$


We check that

$$
\begin{aligned}
\Phi_{1, F}^{\mathcal{U}} & =\Phi_{0, F}^{\left\{V, U_{p+1}\right\}} \circ\left(\Phi_{0, F}^{\mathcal{V}} \oplus \mathrm{id}_{F\left(U_{p+1}\right)}\right) \\
\Phi_{2, F}^{\mathcal{U}} & =\Phi_{1, F}^{\mathcal{V}} \oplus \theta_{1} \\
\Phi_{p+1, F}^{\mathcal{U}} & =\Phi_{p, F}^{\mathcal{U}^{\prime}} \oplus(-1)^{p+2} \theta_{p+1}
\end{aligned}
$$

and, for $j=2, \ldots, p+1$,

$$
\begin{gathered}
\Phi_{j, F}^{\mathcal{U}} \uparrow \bigoplus_{0 \leq i_{1}<\ldots<i_{j} \leq p} F\left(U_{i_{1}, \ldots, i_{j}, p+1}\right)=\Phi_{j, F}^{\mathcal{U}^{\prime}} \oplus(-1)^{j+1} \theta_{j} \\
\Phi_{j, F}^{\mathcal{U}} \uparrow \bigoplus_{0 \leq i_{0}<\ldots<i_{j} \leq p} F\left(U_{i_{0}, \ldots, i_{j}}\right)=\Phi_{j, F}^{\mathcal{V}} .
\end{gathered}
$$

The proof is then completed by means of some diagram chasing which we leave to the reader.

Remark 2.11. Given a topological space $X$ and an abelian group (resp. an $R$ module) we let $C_{q}(X ; G), q=0,1,2, \ldots$, be the group (resp. the $R$-module) of singular $q$-chains in $X$ with coefficients in $G$, see [10, Chapter VII, 1-2]. We recall that the singular homology groups $H_{q}(X ; G)$ are the homology groups of the chain complex

$$
\cdots \stackrel{\partial_{q+1}}{\longrightarrow} C_{q}(X ; G) \stackrel{\partial_{q}}{\longrightarrow} \cdots \stackrel{\partial_{2}}{\longrightarrow} C_{1}(X ; G) \stackrel{\partial_{1}}{\longrightarrow} C_{0}(X ; G) .
$$

The functor $C_{q}(\cdot ; G): \mathrm{O}(X) \rightarrow \mathrm{Ab}$ is not a cosheaf when $q \neq 0$. This drawback is overcome as follows. We let $\mathrm{Sd}: C_{q}(X ; G) \rightarrow C_{q}(X ; G)$ be the barycentric subdivision. This is a natural transformation of the functor $C_{q}(\cdot ; G)$ to itself. We denote by $\mathcal{C}_{q}(\cdot ; G): \mathrm{O}(X) \rightarrow$ Ab the direct limit of the direct system

$$
C_{q}(\cdot ; G) \stackrel{\mathrm{Sd}}{\longrightarrow} C_{q}(\cdot ; G) \stackrel{\mathrm{Sd}}{\longrightarrow} C_{q}(\cdot ; G) \stackrel{\mathrm{Sd}}{\longrightarrow} \cdots
$$

We first notice $\mathrm{Sd}$ is a morphism of chain complexes and that the morphism induced in homology $H_{q}(\mathrm{Sd}): H_{q}(X ; G) \rightarrow H_{q}(X ; G)$ is the identity. Therefore the $H_{q}(X ; G), q=0,1,2, \ldots$, coincide with the homology groups of the chain complex

$$
\cdots \stackrel{\partial_{q+1}}{\longrightarrow} \mathcal{C}_{q}(X ; G) \stackrel{\partial_{q}}{\longrightarrow} \cdots \stackrel{\partial_{2}}{\longrightarrow} \mathcal{C}_{1}(X ; G) \stackrel{\partial_{1}}{\longrightarrow} \mathcal{C}_{0}(X ; G) .
$$

Furthermore we claim that $\mathcal{C}_{q}(\cdot ; G): \mathrm{O}(X) \rightarrow \mathrm{Ab}$ is a cosheaf, as can be checked by applying Proposition 2.10 together with [10, Chapter VII, 8]. 
In the remainder of this paper we will restrict ourselves to Hausdorff topological spaces which are Lindelöf (each cover of $X$ has a subcover which is at most countable). Writing $\operatorname{Cov}^{c}(X)$ for covers of $X$ which are at most countable, it is most obvious that $\operatorname{Cov}^{c}(X)$ is cofinal in $\operatorname{Cov}(X)$ whenever $X$ is Lindelöf. In particular the functors

$$
\text { Lind } \rightarrow \mathrm{Ab}_{\mathrm{c}}\left(\operatorname{resp} . \operatorname{Mod}_{R}\right): X \mapsto \lim _{\mathcal{U} \in \overleftarrow{\operatorname{Cov}}(X)} H_{q}(N(\mathcal{U}) ; G)
$$

and

$$
\text { Lind } \rightarrow \mathrm{Ab}_{\mathrm{c}}\left(\operatorname{resp} . \operatorname{Mod}_{R}\right): X \mapsto \lim _{\mathcal{U} \in \mathrm{Cov}^{c}(X)} H_{q}(N(\mathcal{U}) ; G)
$$

are naturally equivalent (here Lind denotes the category of Hausdorff Lindelöf topological spaces and their continuous maps). Separable paracompact Hausdorff spaces are Lindelöf ([9, Chap. VIII 7.4]), metric spaces are paracompact ([9, Chap. IX 5.3]), and subspaces of second countable spaces are second countable ([9, Chap. VIII 7.2]). Therefore subspaces of separable metric spaces are examples of paracompact Hausdorff Lindelöf spaces.

Definition 2.12. Let $X$ be a topological space, $G$ an abelian group (resp. an $R$-module) and $m \geq 0$ an integer. We say that $X$ is $(H, G, m)$ locally connected (where $H$ refers to singular homology theory) provided the following condition holds. For every $x \in X$ and every open neighborhood $U$ of $x$ there exists an open neighborhood $U^{\prime} \subset U$ of $x$ such that the homomorphism induced by inclusion in reduced singular homology

$$
H_{q}\left(U^{\prime} ; G\right) \rightarrow H_{q}(U ; G)
$$

is trivial for $q=0, \ldots, m+1$.

Example 2.13. It is most obvious that a topological manifold $X$ is $(H, G, m)$ locally connected for each $G$ and $m$ : each $x \in X$ admits arbitrarily small neighborhoods which are (continuously) contractible.

Definition 2.14. Let $G$ be an abelian group (resp. an $R$-module) and $m \geq 0$ be an integer. We let Top ${ }^{L C, G, m}$ denote the category of Hausdorff paracompact Lindelöf topological spaces $X$ which are $(H, G, m)$ locally connected, together with their continuous maps.

The following was proved by S. Mardešić, [13], and extended by G.E. Bredon, [5]. 
Theorem 2.15. Let $G$ be an abelian group (resp. an $R$-module) and let $0 \leq q \leq m$ be integers. The functors

$$
H_{q}: \operatorname{Top}^{L C, G, m} \rightarrow \mathrm{Ab}\left(\operatorname{resp} . \operatorname{Mod}_{R}\right) \text { singular homology }
$$

and

$$
\check{H}_{q}: \operatorname{Top}^{L C, G, m} \rightarrow \mathrm{Ab}\left(\operatorname{resp} . \operatorname{Mod}_{R}\right) \text { Cech homology }
$$

are naturally equivalent.

Proof. Let $X$ be a Hausdorff paracompact Lindelöf $(H, G, m)$ locally connected topological space. With each $\mathcal{U} \in \operatorname{Cov}^{c}(X)$ we associate an augmented double chain complex $\left(A_{*, *}^{\mathfrak{U}} ; L_{*} ; M_{*}^{\mathfrak{U}}\right)$ as follows. For $p, q=0,1,2, \ldots$ we set

$$
\begin{aligned}
A_{p, q}^{\mathcal{U}} & =\bigoplus_{i_{0}<\ldots<i_{p}} \mathcal{C}_{q}\left(U_{i_{0}, \ldots, i_{p}} ; G\right) \\
\Phi_{p, q}^{\mathcal{U}} & =\Phi_{p, \mathcal{C}_{q}}^{\mathcal{U}} \\
\Psi_{p, q}^{\mathcal{U}} & =\oplus \partial q \text { whenever } q \geq 1 \\
L_{q} & =\mathcal{C}_{q}(X ; G) \\
\Psi_{q} & =\partial_{q} \\
M_{q}^{\mathcal{U}} & =S_{q}(N(\mathcal{U}) ; G) \text { (simplicial chains of the nerve) } \\
\Phi_{p}^{\mathcal{U}} & =\partial_{p} .
\end{aligned}
$$

Moreover the augmentation homomorphism

$$
\Psi_{p, 0}^{\mathcal{U}}: \bigoplus_{i_{0}<\ldots<i_{p}} \mathcal{C}_{0}\left(U_{i_{0}, \ldots, i_{p}} ; G\right) \rightarrow S_{p}(N(\mathcal{U}) ; G)
$$

is defined as follows: if $c \in \mathcal{C}_{0}\left(U_{i_{0}, \ldots, i_{p}} ; G\right)$ then we can write $c=\sum g_{k} x_{k}$, $x_{k} \in U_{i_{0}, \ldots, i_{p}}, g_{k} \in G$, where $k$ runs over a finite index set, and we simply let $\Psi_{0, p}^{u}$ be the $p$-chain with carrier $\left\{U_{i_{0}}, \ldots, U_{i_{p}}\right\}$ and coefficient $\sum g_{k}$. It is obvious that $\Psi_{p, 0}^{\mathcal{U}}$ is onto.

This clearly defines an augmented double chain complex. Its rows are exact and $H_{m}\left(L_{*}\right)=H_{m}\left(\bigodot_{*}(X ; G)\right) \cong H_{m}(X ; G)$ (singular homology) according to Remark 2.11. On the other hand $H_{m}\left(M_{*}^{\mathfrak{U}}\right)=H_{m}(N(\mathcal{U}) ; G)$ (simplical homology) so that

$$
\check{H}_{m}(X ; G) \cong \lim _{\mathcal{U} \in \operatorname{Cov}^{c}(X)} H_{m}\left(M_{*}^{\mathcal{u}}\right)
$$


Given $\mathcal{U}, \mathcal{V} \in \operatorname{Cov}^{c}(X)$ such that $\mathcal{U} \preceq \mathcal{V}$ we define $\chi^{\mathcal{U}, \mathcal{V}}$ to be the collection of morphisms of augmented double complexes

$$
\chi^{\mathcal{u}, \mathcal{V}}:\left(A_{*, *}^{\mathcal{u}} ; L_{*} ; M_{*}^{\mathcal{u}}\right) \rightarrow\left(A_{*, *}^{\mathcal{V}} ; L_{*} ; M_{*}^{\mathcal{V}}\right)
$$

associated with refinement projections in the obvious way. The following Claim follows at once from our assumption on $X$ together with Proposition 2.8.

Claim. If $\mathcal{U} \in \operatorname{Cov}^{c}(X)$ then there exists $\mathcal{W} \in \operatorname{Cov}^{c}(X)$ with $\mathfrak{U} \preceq \mathcal{W}$, as well as a morphism of double chain complexes

$$
\chi^{\mathcal{u}, \mathcal{W}}:\left(A_{*, *}^{\mathcal{W}} ; L_{*} ; M_{*}^{\mathcal{W}}\right) \rightarrow\left(A_{*, *}^{\mathcal{U}} ; L_{*} ; M_{*}^{\mathcal{U}}\right)
$$

such that

$$
\chi^{\mathcal{u}, \mathcal{W}}\left(\operatorname{ker} \Psi_{p, q}^{\mathcal{W}}\right) \subset \operatorname{im} \Psi_{p, q+1}^{\mathcal{u}}
$$

for every $p, q=0,1,2, \ldots$.

The Theorem is now a consequence of Theorems 2.4 and 2.5.

\section{Homology in Geometric Measure Theory}

\subsection{Rectifiable and integral currents}

This and the following subsection constitute a short introduction to the vocabulary and notations of Geometric Measure Theory as set forth in [11]. The reader familiar with H. Federer's book can safely skip these subsections ${ }^{2}$.

As usual $m$ and $n$ are integers and $\mathbb{R}^{n}$ is the ambient space. The topological vectorspace of compactly supported smooth differential forms of degree $m$ in $\mathbb{R}^{n}$ is denoted $\mathcal{D}^{m}\left(\mathbb{R}^{n}\right)$ (see $[17$, Chapter 6] for a thorough treatment of the topology of this space). The elements of its dual $\mathcal{D}_{m}\left(\mathbb{R}^{n}\right)$ are called $m d i$ mensional currents. These general objects were studied by G. de Rham ([8]) to show the connections between differential forms and singular chains. Restricted classes of currents (e.g. rectifiable and integral currents) have been introduced by H. Federer and W.H. Fleming in [12] for their relevance in the Calculus of Variations, in particular the problem of Plateau. At about

\footnotetext{
${ }^{2}$ We faithfully follow H. Federer's notations except for the following: given a set $X \subset$ $\mathbb{R}^{n}$ we define $\mathbf{I}_{m}(X)=\mathbf{I}_{m}\left(\mathbb{R}^{n}\right) \cap\{T: \operatorname{spt} T \subset X\}$ and similarly for other collections of currents. Carefully notice that for instance $\mathcal{F}_{m}(X) \neq \mathcal{F}_{m, X}\left(\mathbb{R}^{n}\right)$ (in case $X$ is compact): let e.g. $m=1, n=2$ and let $X=$ Bdry $Z$ be a von Koch snowflake; then $\mathcal{F}_{1, X}\left(\mathbb{R}^{2}\right)=\{0\}$ (recall the Definition of $\mathcal{F}_{m, X}\left(\mathbb{R}^{n}\right),[11,4.1 .24]$ ) because $X$ is $\mathcal{L}^{2}$ negligible and $\left(\mathcal{H}^{1}, 1\right)$ purely unrectifiable, whereas if $T=\partial \mathbf{E}^{2}\left\llcorner Z\right.$ then clearly $0 \neq T \in \mathcal{F}_{1}(X)$.
} 
the same time, H. Whitney developed his theory of flat chains ([20]). One of his primary goals was to obtain a Lebesgue-type theory of integration where the domains of integration are general "distributional" chains and the integrands are differential forms with general nonsmooth coefficients. We are going to quickly review these notions in the spirit of [11].

From now on $0 \leq m \leq n$. We start by observing that 0 dimensional currents $T \in \mathcal{D}_{0}\left(\mathbb{R}^{n}\right)$ are generally called distributions and we save the notation $\mathbf{E}^{n}$ for the following particular $n$ dimensional current in $\mathbb{R}^{n}$ :

$$
\left\langle\mathbf{E}^{n}, \omega\right\rangle=\int_{\mathbb{R}^{n}}\left\langle e_{1} \wedge \ldots \wedge e_{n}, \omega\right\rangle d \mathcal{L}^{n}, \omega \in \mathcal{D}^{n}\left(\mathbb{R}^{n}\right) .
$$

Our next example of an $m$ dimensional current is the oriented simplex $\left[u_{0}, \ldots, u_{m}\right]$ associated with $u_{0}, \ldots, u_{m} \in \mathbb{R}^{n}$ defined as follows:

$$
\left\langle\left[u_{0}, \ldots, u_{m}\right], \omega\right\rangle=\int_{S}\langle\tau, \omega\rangle d \mathcal{H}^{m}, \omega \in \mathcal{D}^{m}\left(\mathbb{R}^{n}\right)
$$

where $S$ is the convex hull of $\left\{u_{0}, \ldots, u_{m}\right\},^{3}$

$$
\tau=\frac{\left(u_{1}-u_{0}\right) \wedge \ldots \wedge\left(u_{m}-u_{0}\right)}{\left|\left(u_{1}-u_{0}\right) \wedge \ldots \wedge\left(u_{m}-u_{0}\right)\right|}
$$

and $\mathcal{H}^{m}$ is the $m$ dimensional Hausdorff measure in $\mathbb{R}^{n}$ (see for instance Appendix B). The additive subgroup of $\mathcal{D}_{m}\left(\mathbb{R}^{n}\right)$ generated by the $m$ dimensional oriented simplexes is denoted $\mathcal{P}_{m}\left(\mathbb{R}^{n}\right)$; its elements are called $m$ dimensional integral polyhedral chains. Similarly, the real vectorsubspace of $\mathcal{D}_{m}\left(\mathbb{R}^{n}\right)$ generated by the $m$ dimensional oriented simplexes is denoted $\mathbf{P}_{m}\left(\mathbb{R}^{n}\right)$; its elements are called $m$ dimensional real polyhedral chains.

The support of a current $T \in \mathcal{D}_{m}\left(\mathbb{R}^{n}\right)$, denoted spt $T$, is the complement of the largest open set $U \subset \mathbb{R}^{n}$ such that $\langle T, \omega\rangle=0$ whenever spt $\omega \subset U$, $\omega \in \mathcal{D}^{m}\left(\mathbb{R}^{n}\right)$. For instance spt $\left[u_{0}, \ldots, u_{m}\right]$ is the convex hull of $\left\{u_{0}, \ldots, u_{m}\right\}$ provided it is not $\mathcal{H}^{m}$ negligible, and empty otherwise. For each set $X \subset \mathbb{R}^{n}$ we also define

$$
\mathcal{P}_{m}(X)=\mathcal{P}_{m}\left(\mathbb{R}^{n}\right) \cap\{T: \operatorname{spt} T \subset X\}
$$

as well as ${ }^{4}$

$$
\mathbf{P}_{m}(X)=\mathbf{P}_{m}\left(\mathbb{R}^{n}\right) \cap\{T: \operatorname{spt} T \subset X\} .
$$

\footnotetext{
${ }^{3}$ The norm $|\cdot|$ on $\wedge^{m} \mathbb{R}^{n}$ is associated with the Euclidean structure of $\mathbb{R}^{n}$ as follows. If $e_{1}, \ldots, e_{n}$ is an orthonormal basis of $\mathbb{R}^{n}$ then we let $\left\langle e_{\lambda(1)} \wedge \ldots \wedge e_{\lambda(m)}, e_{\mu(1)} \wedge \ldots \wedge e_{\mu(m)}\right\rangle=1$ if $\lambda=\mu$ and $=0$ otherwise, where $\lambda, \mu \in \Lambda(n, m)$. Furthermore $|\xi|=\sqrt{\langle\xi, \xi\rangle}, \xi \in \wedge^{m} \mathbb{R}^{n}$.

${ }^{4}$ Notice that in case $X$ is nonempty and compact one has $\mathcal{P}_{m}(X)=\mathcal{P}_{m, X}\left(\mathbb{R}^{n}\right)$ (resp. $\left.\mathbf{P}_{m}(X)=\mathbf{P}_{m, X}\left(\mathbb{R}^{n}\right)\right)$ where the latter is defined in $[11,4.1 .22]$.
} 
Recall that with each differential form $\omega \in \mathcal{D}^{m}\left(\mathbb{R}^{n}\right)$ is associated its exterior derivative $d \omega \in \mathcal{D}^{m+1}\left(\mathbb{R}^{n}\right)$ and its pull-back by $f, f^{\#} \omega \in \mathcal{D}^{m}\left(\mathbb{R}^{\nu}\right)$, whenever $f: \mathbb{R}^{\nu} \rightarrow \mathbb{R}^{n}$ is a smooth proper map. The linear operators $d(\cdot)$ and $f^{\#}(\cdot)$ are continuous, thereby defining by duality the boundary $\partial T \in \mathcal{D}_{m-1}\left(\mathbb{R}^{\nu}\right)$ (in case $m \geq 1$ ) and the push-forward $f_{\#} T \in \mathcal{D}_{m}\left(\mathbb{R}^{n}\right)$ of a current $T \in \mathcal{D}_{m}\left(\mathbb{R}^{\nu}\right)$. One has

$$
\partial\left[u_{0}, \ldots, u_{m}\right]=\sum_{k=0}^{m}(-1)^{k}\left[u_{0}, \ldots, \widehat{u_{k}}, \ldots, u_{m}\right] .
$$

We also notice that in case $f: \mathbb{R}^{\nu} \rightarrow \mathbb{R}^{n}$ is merely smooth (i.e. not necessarily proper) but $T \in \mathcal{D}_{m}\left(\mathbb{R}^{\nu}\right)$ has compact support, then a current $f_{\#} T \in \mathcal{D}_{m}\left(\mathbb{R}^{n}\right)$ is defined by $\left\langle f_{\#} T, \omega\right\rangle:=\left\langle T, \varphi f^{\#} \omega\right\rangle, \omega \in \mathcal{D}^{m}\left(\mathbb{R}^{n}\right)$, where $\varphi \in \mathcal{D}^{0}\left(\mathbb{R}^{\nu}\right)$ is such that $\operatorname{spt} T \subset \operatorname{Int}\{x: \varphi(x)=1\}$. One checks that the definition of $f_{\#} T$ doesn't depend upon the choice of $\varphi$ and that the operator $f_{\#}(\cdot)$ thus defined has the same elementary properties as if $f$ were proper.

The use of smooth differential forms makes it impossible to view a singular simplex " $f_{\#}\left[u_{0}, \ldots, u_{m}\right]$ " as a current because $f$ needs merely be continuous in singular theory. Nevertheless the smoothness of $f$ can be weakened to a Lipschitz condition while preserving a way of making sense of " $f_{\#}\left[u_{0}, \ldots, u_{m}\right]$ " as a current. If $f$ were smooth then

$$
\left\langle f_{\#}\left[u_{0}, \ldots, u_{m}\right], \omega\right\rangle=\left\langle\left[u_{0}, \ldots, u_{m}\right], f^{\#} \omega\right\rangle=\int_{S}\left\langle\tau, f^{\#} \omega\right\rangle d \mathcal{H}^{m} .
$$

In case we merely assume that $\operatorname{Lip} f<\infty$ then applying Rademacher's Theorem (see $[11,3.1 .6]$ ) we infer that $f \uparrow S$ is differentiable in the direction of $\operatorname{span}\left\{u_{1}-u_{0}, \ldots, u_{m}-u_{0}\right\}$ at $\mathcal{H}^{m}$ almost all points of $S$. Moreover $f^{\#} \omega$ is bounded and Borel measurable so that the right hand side of (3.1) makes sense, defining $f_{\#}\left[u_{0}, \ldots, u_{m}\right]$. It can be shown that the new operator $f_{\#}$ behaves similarly to the case of a smooth map $f$, in particular it commutes with the boundary operator, therefore

$$
\partial f_{\#}\left[u_{0}, \ldots, u_{m}\right]=\sum_{k=0}^{m}(-1)^{k} f_{\#}\left[u_{0}, \ldots, \widehat{u_{k}}, \ldots, u_{m}\right] .
$$

One notices that $\partial f_{\#}\left[u_{0}, \ldots, u_{m}\right]$ is "of the same type as" $f_{\#}\left[u_{0}, \ldots, u_{m}\right]-$ the latter is an instance of an integral current.

Assume $T$ is an $m$ dimensional current which is some sort of "Lipschitz chain", as $f_{\#}\left[u_{0}, \ldots, u_{m}\right]$ is, but this time we don't require that $\partial T$ be a "Lipschitz chain" as well. What can $T$ be? Here is an example: if $B \subset \mathbb{R}^{n}$ 
is a Borel set, then the current

$$
\left\langle f_{\#}\left(\left[u_{0}, \ldots, u_{m}\right]\llcorner B), \omega\right\rangle=\int_{S \cap B}\left\langle\tau, f^{\#} \omega\right\rangle d \mathcal{H}^{m}, \omega \in \mathcal{D}^{m}\left(\mathbb{R}^{n}\right)^{5}\right.
$$

is of this type. This is an instance of a rectifiable current, the nomenclature being suggested by the following. Suppose that $f$ is injective. Then a general change of variable Theorem (called the area formula, see [11, 3.2.22]) implies that

$$
\left\langle f_{\#}\left(\left[u_{0}, \ldots, u_{m}\right]\llcorner B), \omega\right\rangle=\int_{M}\left\langle\tau_{M}(y), \omega(y)\right\rangle d \mathcal{H}^{m}(y),\right.
$$

where $M=f(S \cap B)$ is $\left(\mathcal{H}^{m}, m\right)$ rectifiable (see Appendix B) and for $\mathcal{H}^{m}$ almost every $y \in M$,

$$
\tau_{M}(y)=\frac{\left\langle D f(x), u_{1}-u_{0}\right\rangle \wedge \ldots \wedge\left\langle D f(x), u_{m}-u_{0}\right\rangle}{\left|\left\langle D f(x), u_{1}-u_{0}\right\rangle \wedge \ldots \wedge\left\langle D f(x), u_{m}-u_{0}\right\rangle\right|}, x=f^{-1}(y),
$$

(so that, in particular, $\tau_{M}(y)$ is associated with the approximate tangent space of $M$ at $y$ ). Of course if $f$ is not injective then an integral coefficient appears in (3.2), called the degree of $f \uparrow S \cap B$ at $y$, in fact in that case one has

$$
\tau_{M}(y)=\sum_{x \in S \cap B \cap f^{-1}\{y\}} \frac{\left\langle D f(x), u_{1}-u_{0}\right\rangle \wedge \ldots \wedge\left\langle D f(x), u_{m}-u_{0}\right\rangle}{\left|\left\langle D f(x), u_{1}-u_{0}\right\rangle \wedge \ldots \wedge\left\langle D f(x), u_{m}-u_{0}\right\rangle\right|} .
$$

We are now ready to define the rectifiable currents and the integral currents. An $m$ dimensional locally rectifiable current $T$ in $\mathbb{R}^{n}(0 \leq m \leq n)$ is one which can be associated with the following data (see [11, 4.1.28(3)]):

(A) an $\mathcal{H}^{m}$ measurable and countably $\left(\mathcal{H}^{m}, m\right)$ rectifiable set $M$;

(B) an $\mathcal{H}^{m}\left\llcorner M\right.$ locally summable $m$ vectorfield $\eta: \mathbb{R}^{n} \rightarrow \wedge^{m} \mathbb{R}^{n}$ such that for $\mathcal{H}^{m}$ almost every $y \in M$ the number $|\eta(y)|$ is a positive integer and $\eta(y)$ is a simple $m$ vector associated with the approximate tangent space of $M$ at $y$;

in such a way that the following holds

$$
\langle T, \omega\rangle=\int_{M}\langle\eta(y), \omega(y)\rangle d \mathcal{H}^{m}(y), \omega \in \mathcal{D}^{m}\left(\mathbb{R}^{n}\right) .
$$

\footnotetext{
${ }^{5}$ The notation $T\llcorner B$ will be properly introduced at the beginning of the next subsection.
} 
Observe that under conditions (A) and (B) the integral $\langle T, \omega\rangle$ is convergent. Incidentally, these two conditions also imply that $\mathcal{H}^{m}(M \cap C)<\infty$ whenever $C \subset \mathbb{R}^{n}$ is compact. We will often abbreviate the definition of $T$ by writing $T=\mathcal{H}^{m}\llcorner M \wedge \eta$. The integer $|\eta(y)|$ is called the algebraic multiplicity at $y$. (Notice that $y \mapsto|\eta(y)|$ is merely defined up to an $\mathcal{H}^{m}\llcorner M$ negligible set; in order to fix the value of the algebraic multiplicity at each $y$ we may choose the special representative $\Theta^{* m}\left(|\eta| \cdot \mathcal{H}^{m}\llcorner M, y)\right)$. The collection of $m$ dimensional locally rectifiable currents in $\mathbb{R}^{n}$ is an additive subgroup of $\mathcal{D}_{m}\left(\mathbb{R}^{n}\right)$ denoted $\mathcal{R}_{m}^{\text {loc }}\left(\mathbb{R}^{n}\right)$. In case $1 \leq m \leq n$ an $m$ dimensional locally integral current $T$ in $\mathbb{R}^{n}$ is one such that both $T$ and $\partial T$ are locally rectifiable; the 0 dimensional integral currents coincide with the 0 dimensional rectifiable currents. The collection of $m$ dimensional integral currents in $\mathbb{R}^{n}$ is an additive subgroup of $\mathcal{D}_{m}\left(\mathbb{R}^{n}\right)$ denoted $\mathbf{I}_{m}^{\text {loc }}\left(\mathbb{R}^{n}\right)$. An $m$ dimensional current $T \in \mathcal{D}_{m}\left(\mathbb{R}^{n}\right)$ is called rectifiable (resp. integral) if it is locally rectifiable (resp. locally integral) and has compact support; the collection of those is denoted $\mathcal{R}_{m}\left(\mathbb{R}^{n}\right)$ (resp. $\mathbf{I}_{m}\left(\mathbb{R}^{n}\right)$ ). It follows from $[11,4.1 .28(4)]$ that our Definition of rectifiable current (whence also that of integral current) coincides with H. Federer's one $[11,4.1 .24]$. Notice that $\mathbf{I}_{0}\left(\mathbb{R}^{n}\right)=\mathcal{R}_{0}\left(\mathbb{R}^{n}\right)=\mathcal{P}_{0}\left(\mathbb{R}^{n}\right)$ is the set of measures of the form $\sum_{i=1}^{p} \nu_{i} \boldsymbol{\delta}_{a_{i}}$ where $\nu_{i} \in \mathbb{Z}$ and $a_{i} \in \mathbb{R}^{n}$, $i=1, \ldots, p$. Finally, for each set $X \subset \mathbb{R}^{n}$ we set

$$
\mathcal{R}_{m}(X)=\mathcal{R}_{m}\left(\mathbb{R}^{n}\right) \cap\{T: \operatorname{spt} T \subset X\}
$$

and

$$
\mathbf{I}_{m}(X)=\mathbf{I}_{m}\left(\mathbb{R}^{n}\right) \cap\{T: \operatorname{spt} T \subset X\} .
$$

\subsection{Real and integral flat chains}

Let $U \subset \mathbb{R}^{n}$ be open. The mass of a current $T \in \mathcal{D}_{m}\left(\mathbb{R}^{n}\right)$ in $U$ is defined as follows ${ }^{6}$ :

$$
\begin{aligned}
\mathbf{M}_{U}(T)=\sup \left\{\langle T, \omega\rangle: \omega \in \mathcal{D}^{m}\left(\mathbb{R}^{n}\right),\right. & \text { spt } \omega \subset U \\
& \text { and }\|\omega(x)\| \leq 1 \text { for every } x \in U\} .
\end{aligned}
$$

For instance if $T=\mathcal{H}^{m}\left\llcorner M \wedge \eta \in \mathcal{R}_{m}^{\text {loc }}\left(\mathbb{R}^{n}\right)\right.$ then

$$
\mathbf{M}_{U}(T)=\int_{M \cap U}|\eta| d \mathcal{H}^{m}<\infty
$$

thus the mass in $U$ of a locally rectifiable $m$ dimensional current is its $m$ dimensional area in $U$ weighted by its algebraic multiplicity. If $U=\mathbb{R}^{n}$ we simply write $\mathbf{M}(T)$ instead of $\mathbf{M}_{\mathbb{R}^{n}}(T)$, and we call this the mass of $T$.

\footnotetext{
${ }^{6}\|\omega\|=\sup \left\{\langle\xi, \omega\rangle: \xi \in \wedge^{m} \mathbb{R}^{n}\right.$ is simple and $\left.|\xi| \leq 1\right\}$.
} 
In general the mass can be locally finite or infinite. In view of the RieszMarkov Theorem (see e.g. [11, 2.5.12-13]) if $T \in \mathcal{D}_{m}\left(\mathbb{R}^{n}\right)$ has finite mass in each bounded open set $U$ then there exists a Radon measure $\|T\|$ on $\mathbb{R}^{n}$ and a locally $\|T\|$ summable $m$ vectorfield $\vec{T}: \mathbb{R}^{n} \rightarrow \wedge^{m} \mathbb{R}^{n}$ such that $|\vec{T}|=1\|T\|$ almost everywhere and $\langle T, \omega\rangle=\int_{\mathbb{R}^{n}}\langle\omega, \vec{T}\rangle d\|T\|$. For example if $T=\mathcal{H}^{m}\left\llcorner M \wedge \eta\right.$ then $\|T\|=|\eta| \cdot \mathcal{H}^{m}\left\llcorner M\right.$ and $\vec{T}=\eta|\eta|^{-1}$. If $T \in \mathcal{D}_{m}\left(\mathbb{R}^{n}\right)$ has finite local mass in each bounded open subset of $\mathbb{R}^{n}$, and if $A \subset \mathbb{R}^{n}$ is Borel, then we define $T\left\llcorner A:=\|T\|\left\llcorner A \wedge \vec{T}\right.\right.$, i.e. $\left\langle T\llcorner A, \omega\rangle=\int_{A}\langle\vec{T}, \omega\rangle d\|T\|\right.$, $\omega \in \mathcal{D}^{m}\left(\mathbb{R}^{n}\right)$. We observe $T\left\llcorner A \in \mathcal{D}_{m}\left(\mathbb{R}^{n}\right)\right.$, in fact $\mathbf{M}_{U}(T\llcorner A) \leq\|T\|(A \cap U)$ whenever $U \subset \mathbb{R}^{n}$ is open.

An $m$ dimensional locally normal current $T \in \mathcal{D}_{m}\left(\mathbb{R}^{n}\right)$ is one such that for each open bounded set $U \subset \mathbb{R}^{n}$ one has $\mathbf{M}_{U}(T)+\mathbf{M}_{U}(\partial T)<\infty$ in case $m \geq 1$, or simply $\mathbf{M}_{U}(T)<\infty$ in case $m=0$. For instance locally integral currents are locally normal. The real vectorsubspace of $\mathcal{D}_{m}\left(\mathbb{R}^{n}\right)$ consisting of locally normal currents is denoted $\mathbf{N}_{m}^{\text {loc }}\left(\mathbb{R}^{n}\right)$. A normal current is a locally normal current having compact support. The subspace of $\mathbf{N}_{m}^{\text {loc }}\left(\mathbb{R}^{n}\right)$ consisting of normal currents is denoted $\mathbf{N}_{m}\left(\mathbb{R}^{n}\right)$.

From the discussion of the preceding subsection we recall that there exists an operator $f_{\#}(\cdot)$ defined on a certain restricted class of currents (namely we treated the case of the polyhedral chains) in case $f$ is merely Lipschitzian. Furthemore formula (3.2) defining $f_{\#}\left[u_{0}, \ldots, u_{m}\right]$ implies that

$$
f_{j \#}\left[u_{0}, \ldots, u_{m}\right] \rightarrow f_{\#}\left[u_{0}, \ldots, u_{m}\right] \text { weakly as } j \rightarrow \infty
$$

provided $f, f_{1}, f_{2}, \ldots$ are Lipschitzian and meet the following conditions:

(A) $\sup \left\{\operatorname{Lip} f_{j}: j=1,2, \ldots\right\}<\infty$;

(B) $f_{j} \rightarrow f$ uniformly as $j \rightarrow \infty$;

(C) $D\left(f_{j} \uparrow S\right)(x) \rightarrow D(f \uparrow S)(x)$ as $j \rightarrow \infty$ for $\mathcal{H}^{m}$ almost every $x \in S$ (where, as before, $S$ is convex hull of $\left\{u_{0}, \ldots, u_{m}\right\}$ ).

Given a Lipschitzian $f$, the mollified functions $f_{j}=\Phi_{j} * f$ are smooth and verify these conditions. This is of course an invitation to define the operator $f_{\#}(\cdot)$ as a limit in some sense of the operators $f_{j \#}(\cdot)$. We will now introduce a complete topology which makes $f_{j \#} T, j=1,2, \ldots$, into a Cauchy sequence in case $T$ is normal and $f_{j}, j=1,2, \ldots$, are smooth and verify only conditions (A) and (B).

To this end we first state the homotopy formula for currents (see e.g. [8, $\S 14]$ ). Assume that $h: \mathbb{R} \times \mathbb{R}^{n} \rightarrow \mathbb{R}^{\nu}$ is smooth and let $f(x)=h(0, x)$, $g(x)=h(1, x), x \in \mathbb{R}^{n}$. Then for each $T \in \mathcal{D}_{m}\left(\mathbb{R}^{n}\right)$ with compact support we have

$$
g_{\#} T-f_{\#} T= \begin{cases}\partial h_{\#}([0,1] \times T)+h_{\#}([0,1] \times \partial T) & \text { if } m \geq 1 \\ \partial h_{\#}([0,1] \times T) & \text { if } m=0\end{cases}
$$


Next we turn to the particular case when $h$ is the affine homotopy from $f$ to $g$ :

$$
h(t, x)=(1-t) f(x)+\operatorname{tg}(x) .
$$

A straightforward computation involving only the definition of push-forward and cartesian product yields

$$
\mathbf{M}\left(h_{\#}([0,1] \times Z)\right) \leq \max \{\operatorname{Lip} f, \operatorname{Lip} g\}^{q} \int_{\mathbb{R}^{n}}|f-g| d\|Z\|
$$

whenever $Z \in \mathcal{D}_{q}\left(\mathbb{R}^{n}\right), q=0, \ldots, n$ and $\mathbf{M}(Z)<\infty$. We now define the flat semi-norm of $T \in \mathcal{D}_{m}\left(\mathbb{R}^{n}\right)$ in an open set $U \subset \mathbb{R}^{n}$ as follows:

$$
\begin{aligned}
& \mathbf{F}_{U}(T)=\inf \left\{\mathbf{M}_{U}(R)+\mathbf{M}_{U}(S): T=R+\partial S, R \in \mathcal{D}_{m}\left(\mathbb{R}^{n}\right)\right. \\
& \left.\quad \text { and } S \in \mathcal{D}_{m+1}\left(\mathbb{R}^{n}\right)\right\} .
\end{aligned}
$$

It may happen that $\mathbf{F}_{U}(T)=\infty$, nevertheless $\mathbf{F}_{U}(T) \leq \mathbf{M}_{U}(T)$ and equality holds when $m=n$. One easily checks that if $C \subset U$ is compact then the restriction of $\mathbf{F}_{U}$ to $\mathcal{D}_{m}\left(\mathbb{R}^{n}\right) \cap\left\{T: \operatorname{spt} T \subset C\right.$ and $\left.\mathbf{F}_{U}(T)<\infty\right\}$ makes it into a Banach space. Therefore $\mathcal{D}_{m}\left(\mathbb{R}^{n}\right) \cap\left\{T: \mathbf{F}_{U}(T)<\infty\right.$ for every bounded open $\left.U \subset \mathbb{R}^{n}\right\}$ becomes a Fréchet space when endowed with the locally convex vector topology generated by the semi-norms $\mathbf{F}_{U}, U \subset \mathbb{R}^{n}$ bounded and open. We will call this the local flat topology.

The importance of the flat semi-norms stems from the following estimate. If $T \in \mathbf{N}_{m}\left(\mathbb{R}^{n}\right), f, g: \mathbb{R}^{n} \rightarrow \mathbb{R}^{\nu}$ are smooth and if the convex hull of $f(\operatorname{spt} T) \cup g(\operatorname{spt} T)$ is contained in an open set $U \subset \mathbb{R}^{\nu}$ then

$$
\mathbf{F}_{U}\left(f_{\#} T-g_{\#} T\right) \leq \lambda^{m} \int_{\mathbb{R}^{n}}|f-g| d\|T\|+\lambda^{m-1} \int_{\mathbb{R}^{n}}|f-g| d\|\partial T\|
$$

where $\lambda=\max \{\operatorname{Lip} f, \operatorname{Lip} g\}$. In particular, if $f_{j}, j=1,2, \ldots$, verify conditions (A) and (B) above and if $T \in \mathbf{N}_{m}\left(\mathbb{R}^{n}\right)$ is such that $f_{j}(\operatorname{spt} T) \subset C \subset U$, $j=1,2, \ldots$, for some open set $U \subset \mathbb{R}^{n}$ and compact set $C \subset U$, then $f_{j \#} T$, $j=1,2, \ldots$, is an $\mathbf{F}_{U}$ Cauchy sequence therefore converging to a current denoted $f_{\#} T$ which belongs to the $\mathbf{F}_{U}$ completion of $\mathbf{N}_{m}\left(\mathbb{R}^{\nu}\right) \cap\{S: \operatorname{spt} S \subset C\}$ (moreover $f_{\#} T$ readily doesn't depend upon the choice of a particular sequence $\left.f_{j}, j=1,2, \ldots\right)$. We say that a current $T \in \mathcal{D}_{m}\left(\mathbb{R}^{n}\right)$ is an $m$ dimensional locally flat chain if it belongs to the completion of $\mathbf{N}_{m}^{\text {loc }}\left(\mathbb{R}^{n}\right)$ in the local flat topology. The real vectorsubspace of $\mathcal{D}_{m}\left(\mathbb{R}^{n}\right)$ consisting of locally flat chains is denoted $\mathbf{F}_{m}^{\text {loc }}\left(\mathbb{R}^{n}\right)$. If $T \in \mathbf{F}_{m}^{\text {loc }}\left(\mathbb{R}^{n}\right)$ and $\operatorname{spt} T$ is compact then we say that $T$ is an $m$ dimensional flat chain and we let $\mathbf{F}_{m}\left(\mathbb{R}^{n}\right)$ be the vectorspace consisting of those currents. We have just defined a linear operator $f_{\#}: \mathbf{N}_{m}\left(\mathbb{R}^{n}\right) \rightarrow \mathbf{F}_{m}\left(\mathbb{R}^{\nu}\right)$ in case $f: \mathbb{R}^{n} \rightarrow \mathbb{R}^{\nu}$ is 
Lipschitzian. This operator inherits in the limit the elementary properties of the operators $\left(\Phi_{j} * f\right)_{\#}(\cdot), j=1,2, \ldots$, in particular it commutes with $\partial$. Therefore $\mathbf{F}_{U}\left(f_{\#} T\right) \leq \mathbf{F}_{f^{-1}(U)}(T)$ whenever $T \in \mathbf{N}_{m}\left(\mathbb{R}^{n}\right)$ and $U \subset \mathbb{R}^{\nu}$ is open. Hence $f_{\#}$ extends to a linear operator $f_{\#}: \mathbf{F}_{m}\left(\mathbb{R}^{n}\right) \rightarrow \mathbf{F}_{m}\left(\mathbb{R}^{\nu}\right)$. Referring to the area formula (see $[11,3.2 .22]$ ) it is possible to show that $f_{\#}\left(\mathcal{R}_{m}\left(\mathbb{R}^{n}\right)\right) \subset \mathcal{R}_{m}\left(\mathbb{R}^{\nu}\right)$ and in turn $f_{\#}\left(\mathbf{I}_{m}\left(\mathbb{R}^{n}\right)\right) \subset \mathbf{I}_{m}\left(\mathbb{R}^{\nu}\right)$.

We now turn to defining integral flat chains. We let

$$
\mathcal{F}_{m}^{\text {loc }}\left(\mathbb{R}^{n}\right)=\left\{R+\partial S: R \in \mathcal{R}_{m}^{\text {loc }}\left(\mathbb{R}^{n}\right) \text { and } S \in \mathcal{R}_{m+1}^{\text {loc }}\left(\mathbb{R}^{n}\right)\right\},
$$

and we call $m$ dimensional locally integral flat chains the elements of $\mathcal{F}_{m}^{\text {loc }}\left(\mathbb{R}^{n}\right)$. An $m$ dimensional integral flat chain is a current $T \in \mathcal{F}_{m}^{\text {loc }}\left(\mathbb{R}^{n}\right)$ such that spt $T$ is compact; these form an additive subgroup of $\mathcal{D}_{m}\left(\mathbb{R}^{n}\right)$ denoted $\mathcal{F}_{m}\left(\mathbb{R}^{n}\right)$. Given $T \in \mathcal{F}_{m}^{\text {loc }}\left(\mathbb{R}^{n}\right)$ and an open set $U \subset \mathbb{R}^{n}$ we define the local integral flat norm of $T$ in $U$ as follows:

$$
\begin{aligned}
\mathcal{F}_{U}(T)=\inf \left\{\mathbf{M}_{U}(R)+\mathbf{M}_{U}(S): T=R+\partial S, R\right. & \in \mathcal{R}_{m}^{\text {loc }}\left(\mathbb{R}^{n}\right) \\
& \text { and } \left.S \in \mathcal{R}_{m+1}^{\text {loc }}\left(\mathbb{R}^{n}\right)\right\} .
\end{aligned}
$$

As usual we write $\mathcal{F}(T)$ instead of $\mathcal{F}_{\mathbb{R}^{n}}(T)$. We notice that $\mathcal{F}_{m}^{\text {loc }}\left(\mathbb{R}^{n}\right) \subset$ $\mathbf{F}_{m}^{\text {loc }}\left(\mathbb{R}^{n}\right)$. It also follows from the previous paragraph that if $T \in \mathcal{F}_{m}\left(\mathbb{R}^{n}\right)$ and $f: \mathbb{R}^{n} \rightarrow \mathbb{R}^{\nu}$ is Lipschitzian then $f_{\#} T \in \mathcal{F}_{m}\left(\mathbb{R}^{\nu}\right)$.

We now intend to show that every integral flat chain $T \in \mathcal{F}_{m}\left(\mathbb{R}^{n}\right)$ can be approximated in the $\mathcal{F}_{U}$ semi-norms by integral polyhedral chains. This will be done in two steps, the latter being the important "strong approximation Theorem" for integral currents.

Lemma 3.1. If $T \in \mathcal{R}_{m}\left(\mathbb{R}^{n}\right)$ and $\varepsilon>0$ there exists $T^{\prime} \in \mathbf{I}_{m}\left(\mathbb{R}^{n}\right)$ such that $\mathbf{M}\left(T-T^{\prime}\right)<\varepsilon$.

Proof. If $m=0$ the conclusion trivially holds; we henceforth assume $m \geq 1$. According to $[11,4.1 .28(3)]$ there exist an open set $Z \subset \mathbb{R}^{m}$, a compact subset $A \subset Z$ and a Lipschitzian map $f: Z \rightarrow \mathbb{R}^{n}$ such that

$$
\mathbf{M}\left(T-f_{\#}\left(\mathbf{E}^{m}\llcorner A)\right)<\varepsilon / 2 .\right.
$$

Choose $P \in \mathcal{P}_{m}(\mathbb{Z})$ such that

$$
\mathbf{M}\left(P-\mathbf{E}^{m}\llcorner A)<\varepsilon(\operatorname{Lip} f)^{-m} / 2\right.
$$

- e.g. $P=\sum_{D \in \mathcal{D}} \mathbf{E}^{m} \mathbf{L} D$ for some suitable finite family $\mathcal{D}$ of dyadic cubes. Now

$$
f_{\#} P \in \mathbf{I}_{m}\left(\mathbb{R}^{n}\right) \quad \text { and } \quad \mathbf{M}\left(f_{\#} P-f_{\#}\left(\mathbf{E}^{m}\llcorner A)\right)<\varepsilon / 2 .\right.
$$

We conclude that $\mathbf{M}\left(T-f_{\#} P\right)<\varepsilon$. 
Theorem 3.2. Whenever $T \in \mathbf{I}_{m}\left(\mathbb{R}^{n}\right)$ and $\varepsilon>0$ there exist $P \in \mathcal{P}_{m}\left(\mathbb{R}^{n}\right)$ and a diffeomorphism $f: \mathbb{R}^{n} \rightarrow \mathbb{R}^{n}$ of class $C^{1}$ such that:

(A) $\operatorname{spt} P \subset \mathbf{B}(\operatorname{spt} T, \varepsilon)$;

(B) $\mathbf{N}\left(P-f_{\#} T\right)<\varepsilon$;

(C) $\max \left\{\operatorname{Lip} f, \operatorname{Lip} f^{-1}\right\}<1+\varepsilon$;

(D) $|f(x)-x|<\varepsilon$ for $x \in \mathbb{R}^{n}$;

(E) $f(x)=x$ whenever $x \in \mathbb{R}^{n}$ and $\operatorname{dist}(x$, spt $T)>\varepsilon$.

For a proof of this Theorem see [11, 4.2.20]. In a forthcoming paper [7] we will prove an analogous Theorem for compact rectifiable surfaces which do not have the structure of a current.

Corollary 3.3. Whenever $T \in \mathbf{I}_{m}\left(\mathbb{R}^{n}\right)$ and $\varepsilon>0$ there exists $P \in \mathcal{P}_{m}\left(\mathbb{R}^{n}\right)$ such that $\mathcal{F}(T-P)<\varepsilon$.

Proof. Choose $\eta>0$ and let $P$ and $f$ be associated with $T$ and $\eta$ as in Theorem 3.2. We observe that the homotopy formula (3.3) holds when $f$ and $g$ are Lipschitzian and $T \in \mathbf{F}_{m}\left(\mathbb{R}^{n}\right)$. This together with the estimate (3.4) and the Definition of $\mathcal{F}$ yields $\mathcal{F}\left(T-f_{\#} T\right)<2(1+\eta)^{m} \eta \mathbf{N}(T)$. It also follows from Theorem 3.2 that $\mathcal{F}\left(P-f_{\#} T\right) \leq \mathbf{M}\left(P-f_{\#} T\right)<\eta$. Therefore $\mathcal{F}(T-P)<2(1+\eta)^{m} \eta \mathbf{N}(T)+\eta<\varepsilon$ provided $\eta$ is small enough.

Proposition 3.4. Whenever $T \in \mathcal{F}_{m}\left(\mathbb{R}^{n}\right)$ and $\varepsilon>0$ there exists $P \in$ $\mathcal{P}_{m}\left(\mathbb{R}^{n}\right)$ such that $\mathcal{F}(T-P)<\varepsilon$.

Proof. Choose $R \in \mathcal{R}_{m}\left(\mathbb{R}^{n}\right)$ and $S \in \mathcal{R}_{m+1}\left(\mathbb{R}^{n}\right)$ such that $T=R+\partial S$. Since spt $T$ is compact there exists $r>0$ so that spt $T \subset \mathbf{U}(0, r)$. Let $f: \mathbb{R}^{n} \rightarrow \mathbb{R}^{n}$ be the nearest point projection onto $\mathbf{B}(0, r)$ and put $R^{\prime}=f_{\#} R$ and $S^{\prime}=$ $f_{\#} S$. The clearly $T=R^{\prime}+\partial S^{\prime}$ and $R^{\prime} \in \mathcal{R}_{m}\left(\mathbb{R}^{n}\right)$ and $S^{\prime} \in \mathcal{R}_{m+1}\left(\mathbb{R}^{n}\right)$. Now refer to Lemma 3.1 to pick $R^{\prime \prime} \in \mathbf{I}_{m}\left(\mathbb{R}^{n}\right)$ and $S^{\prime \prime} \in \mathbf{I}_{m+1}\left(\mathbb{R}^{n}\right)$ in order that $\mathbf{M}\left(R^{\prime}-R^{\prime \prime}\right)<\varepsilon / 4$ and $\mathbf{M}\left(S^{\prime}-S^{\prime \prime}\right)<\varepsilon / 4$. Letting $T^{\prime \prime}=R^{\prime \prime}+\partial S^{\prime \prime} \in \mathbf{I}_{m}\left(\mathbb{R}^{n}\right)$ we readily check that $\mathcal{F}\left(T-T^{\prime \prime}\right)<\varepsilon / 2$. Next we infer from Corollary 3.3 that there exist $P^{\prime \prime} \in \mathcal{P}_{m}\left(\mathbb{R}^{n}\right)$ and $Q^{\prime \prime} \in \mathcal{P}_{m+1}\left(\mathbb{R}^{n}\right)$ such that $\mathcal{F}\left(R^{\prime \prime}-P^{\prime \prime}\right)<\varepsilon / 2$ and $\mathcal{F}\left(S^{\prime \prime}-Q^{\prime \prime}\right)<\varepsilon / 2$. Finally we define $P=P^{\prime \prime}+\partial Q^{\prime \prime} \in \mathcal{P}_{m}\left(\mathbb{R}^{n}\right)$ and we check that $\mathcal{F}(T-P) \leq \mathcal{F}\left(T-T^{\prime \prime}\right)+\mathcal{F}\left(R^{\prime \prime}-P^{\prime \prime}\right)+\mathcal{F}\left(\partial\left(S^{\prime \prime}-Q^{\prime \prime}\right)\right)<\varepsilon$. 


\subsection{Homology theories and how they compare with Čech's}

In this subsection we recall the Definition of the integral flat homology groups introduced in $[11,4.4 .1]$ and we define likewise the integral rectifiable homology groups. We show these are homology theories in the sense of Eilenberg and Steenrod on the category of pairs $(X, A)$ with $A \subset X \subset \mathbb{R}^{n}$ for some integer $n$, and their locally Lipschitzian maps. We notice that the Excision Axiom is verified without restriction on the pair $(X, A)$ (compare with $[11,4.4 .1])$. In the next subsection we provide various examples showing these theories don't coincide, nor do they compare to either singular or Čech homology. Nevertheless, applying the general comparison Theorem proved the preceding section, we are able to establish that the four homology theories are naturally equivalent on certain restricted classes of pairs, e.g. Lipschitzian submanifolds of Euclidean space.

We consider the category $\mathcal{A}$ defined as follows. Its objects are the pairs $(X, A)$ of subsets $A \subset X \subset \mathbb{R}^{n}$ of some Euclidean space. The morphisms $f:(X, A) \rightarrow(Y, B)$ are the locally Lipschitzian maps $f: X \rightarrow Y$ such that $f(A) \subset B$. This is clearly an admissible category for homology theory in the sense of $[10$, Definition p.5]. Let $(X, A)$ be an object of $\mathcal{A}$. Clearly the boundary operator $\partial$ maps $\mathcal{F}_{m}(X)$ in $\mathcal{F}_{m-1}(X)$ and the kernel of the composition

$$
\mathcal{F}_{m}(X) \stackrel{\partial}{\longrightarrow} \mathcal{F}_{m-1}(X) \stackrel{p}{\longrightarrow} \frac{\mathcal{F}_{m-1}(X)}{\mathcal{F}_{m-1}(A)}
$$

contains $\mathcal{F}_{m}(A)$. Therefore on letting $\mathcal{F}_{m}(X, A)$ denote the quotient group $\frac{\mathcal{F}_{m}(X)}{\mathcal{F}_{m}(A)}$ we have a well defined chain complex

$$
\mathcal{F}_{m}(X, A) \stackrel{p \circ \partial}{\longrightarrow} \mathcal{F}_{m-1}(X, A), m \geq 1 \text {. }
$$

The corresponding homology groups are denoted $\mathbf{H}_{m}(X, A)$ and called the $m$ dimensional integral flat homology groups of the pair $(X, A)$. As usual we write $\mathbf{H}_{m}(X)$ as an abbreviation for $\mathbf{H}_{m}(X, \emptyset)$. In case $f:(X, A) \rightarrow$ $(Y, B)$ is a morphism and $T \in \mathcal{F}_{m}(X)$ we recall that there is a well-defined homomorphism $f_{\#}: \mathcal{F}_{m}(X) \rightarrow \mathcal{F}_{m}(Y)$ which commutes with the boundary operator and such that spt $f_{\#} T \subset f(\operatorname{spt} T)$. Moreover $(g \circ f)_{\#}=g_{\#} \circ f_{\#}$. We now see how $f$ induces a morphism of chain complexes

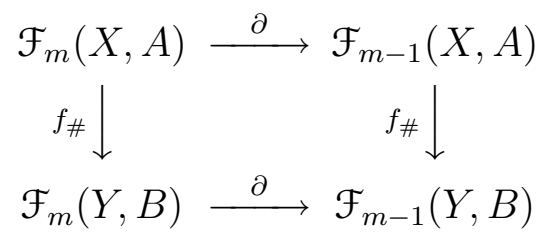

and, in turn, homomorphisms $\mathbf{H}_{m}(f): \mathbf{H}_{m}(X, A) \rightarrow \mathbf{H}_{m}(Y, B), m \geq 0$. 
Replacing the groups of integral flat chains $\mathcal{F}_{m}(X)$ with the groups of integral currents $\mathbf{I}_{m}(X)$ in the above construction, we define the groups $\overline{\mathbf{H}}_{m}(X, A)$ and homomorphisms $\overline{\mathbf{H}}_{m}(f): \overline{\mathbf{H}}_{m}(X, A) \rightarrow \overline{\mathbf{H}}(Y, B)$ associated with an object $(X, A)$ and a morphism $f:(X, A) \rightarrow(Y, B)$ of the category $\mathcal{A}$. We call $\overline{\mathbf{H}}_{m}(X, A)$ the $m$ dimensional integral rectifiable homology group of the pair $(X, A)$.

We now turn to proving that both $\mathbf{H}$ and $\overline{\mathbf{H}}$ verify the axioms of Eilenberg and Steenrod (see [10, Chap. I]). The following Lemma will be useful when checking that the Excision Axiom holds.

Lemma 3.5. Let $T \in \mathcal{F}_{m}\left(\mathbb{R}^{n}\right)$ (resp. $T \in \mathbf{I}_{m}\left(\mathbb{R}^{n}\right)$ ) and let $u: \mathbb{R}^{n} \rightarrow \mathbb{R}$ be Lipschitzian. For $\mathcal{L}^{1}$ almost every $r \in \mathbb{R}$ there exist $T_{u, r}^{-}, T_{u, r}^{+} \in \mathcal{F}_{m}\left(\mathbb{R}^{n}\right)$ (resp. $T_{u, r}^{-}, T_{u, r}^{+} \in \mathbf{I}_{m}\left(\mathbb{R}^{n}\right)$ ) such that

$$
T=T_{u, r}^{-}+T_{u, r}^{+}
$$

and

$$
\begin{aligned}
& \operatorname{spt} T_{u, r}^{-} \subset \operatorname{spt} T \cap\{x: u(x) \leq r\} \\
& \operatorname{spt} T_{u, r}^{+} \subset \operatorname{spt} T \cap\{x: u(x) \geq r\}
\end{aligned}
$$

Proof. We prove the Lemma in case $T \in \mathcal{F}_{m}\left(\mathbb{R}^{n}\right)$ and we will indicate at the end which statements from [11] to refer to for the case $T \in \mathbf{I}_{m}\left(\mathbb{R}^{n}\right)$. We start by observing that if $T \in \mathcal{P}_{m}\left(\mathbb{R}^{n}\right)$ then $\mathbf{M}(T)<\infty$ and we can define

$$
\begin{aligned}
& T_{u, r}^{-}=T\llcorner\{x: u(x) \leq r\} \\
& T_{u, r}^{+}=T\llcorner\{x: u(x)>r\} .
\end{aligned}
$$

We readily check these currents verify the conclusions of the Lemma.

Next we claim that for $P \in \mathcal{P}_{m}\left(\mathbb{R}^{n}\right)$ the function

$$
\mathbb{R} \rightarrow \mathbb{R}: r \mapsto \mathcal{F}(P\llcorner\{x: u(x) \leq r\})
$$

is Borel. Indeed for $r \in \mathbb{R}$ and $h>0$ one has

$$
\begin{aligned}
\mid \mathcal{F}(P\llcorner\{x & : u(x) \leq r\})-\mathcal{F}(P\llcorner\{x: u(x) \leq r-h\}) \mid \\
& \leq \mathcal{F}(P\llcorner\{x: r-h<u(x) \leq r\}) \\
& \leq\|P\|\{x: r-h<u(x) \leq r\},
\end{aligned}
$$

and since $\|P\|\{x: u(x)=r\}=0$ for all but countably many $r \in \mathbb{R}$ we conclude that the function defined by (3.6) is Borel.

Next we infer from $[11,4.2 .1]$ that for $P$ as above,

$$
\int_{a}^{b} \mathcal{F}\left(P\llcorner\{x: u(x) \leq r\}) d \mathcal{L}^{1}(r) \leq(b-a+\operatorname{Lip}(u)) \mathcal{F}(P)\right.
$$

for every $-\infty<a<b<+\infty$. 
Now given $T \in \mathcal{F}_{m}\left(\mathbb{R}^{n}\right)$ we infer from Proposition 3.4 that there exist $P_{j} \in \mathcal{P}_{m}\left(\mathbb{R}^{n}\right), j=1,2, \ldots$, such that $\mathcal{F}\left(T-P_{j}\right) \leq 2^{-j-1}$ for each $j$. We may also assume that spt $P_{j} \subset B, j=1,2, \ldots$, where $B$ is some Euclidean ball containing the support of $T$. From (3.6) applied with $P=P_{j}-P_{k}$ we infer that

$$
\int_{a}^{b} \mathcal{F}\left(\left(P_{j}\right)_{u, r}^{-}-\left(P_{k}\right)_{u, r}^{-}\right) d \mathcal{L}^{1}(r) \leq(b-a+\operatorname{Lip}(u)) 2^{-j}
$$

whenever $k \geq j$. Therefore, on letting

$$
g_{j}(r)=\sum_{k=j}^{\infty} \mathcal{F}\left(\left(P_{k+1}\right)_{u, r}^{-}-\left(P_{k}\right)_{u, r}^{-}\right),
$$

we deduce from (3.8) that

$$
\int_{a}^{b} g_{j} d \mathcal{L}^{1} \leq(b-a+\operatorname{Lip}(u)) 2^{-j+1} .
$$

According to Fatou's Lemma we infer that $g_{j}(r) \downarrow 0$ as $j \rightarrow \infty$ for $\mathcal{L}^{1}$ almost every $r \in \mathbb{R}$. This clearly implies, for those $r$ 's, that $\left(P_{1}\right)_{u, r}^{-},\left(P_{2}\right)_{u, r}^{-}, \ldots$ is an $\mathcal{F}$ Cauchy sequence. Let $T_{u, r}^{-} \in \mathcal{F}_{m, K}\left(\mathbb{R}^{n}\right)$ be its limit, and $T_{u, r}^{+}=T-T_{u, r}^{-}$ (it follows from the $\mathbf{M}$ completeness of $\mathcal{R}_{m}(B)$ and $\mathcal{R}_{m+1}(B)$ that $\mathcal{F}_{m}(B)$ is $\mathcal{F}$ complete). These currents readily verify the conclusions of the Lemma.

In case $T \in \mathbf{I}_{m}\left(\mathbb{R}^{n}\right)$ we refer to $[11,4.2 .1$ and 4.2.16(2)] to guarantee that $T\left\llcorner\{x: u(x) \leq r\} \in \mathbf{I}_{m}\left(\mathbb{R}^{n}\right)\right.$ for $\mathcal{L}^{1}$ almost every $r \in R$.

Remark 3.6. We show that "For $\mathcal{L}^{1}$ almost every $r \in \mathbb{R} \ldots$... cannot be replaced by "For every $r \in \mathbb{R}$..." in the conclusion of Lemma 3.5. Consider

$$
S=\sum_{j=1}^{\infty}\left[\left(j^{-1}, j^{-2}\right),\left(j^{-1},-j^{-2}\right)\right] \in \mathcal{R}_{1}\left(\mathbb{R}^{2}\right)
$$

and $T=\partial S \in \mathcal{F}_{0}\left(\mathbb{R}^{2}\right)$, as well as $u: \mathbb{R}^{2} \rightarrow \mathbb{R}$ defined by $u\left(x_{1}, x_{2}\right)=x_{2}$. One checks that there are no distributions $T_{u, 0}^{-}, T_{u, 0}^{+} \in \mathcal{D}_{0}\left(\mathbb{R}^{2}\right)$ verifying the conclusions of the Lemma for $T, u$ and $r=0$. On the other hand if $A \subset \mathbb{R}^{n}$ is a bounded non $\mathcal{L}^{n}$ negligible set with infinite perimeter (that is if $\mathbf{M}\left(\partial \mathbf{E}^{n}\llcorner A)=\infty\right.$, for instance if $A \subset \mathbb{R}$ is a non $\mathcal{L}^{1}$ negligible topological Cantor set or $A \subset \mathbb{R}^{2}$ is a snowflake), then one checks that the conclusions of the Lemma with $T_{u, r}^{-}, T_{u, r}^{+} \in \mathbf{I}_{n}\left(\mathbb{R}^{n}\right)$ cannot be verified for $T=\mathbf{E}^{n} \mathbf{L} A$, $u=\operatorname{dist}(\cdot, A), x \in \mathbb{R}^{n}$, and $r=0$.

Proposition 3.7. The integral flat $\mathbf{H}$ and integral rectifiable $\overline{\mathbf{H}}$ homology theories defined on the category $\mathcal{A}$ verify the axioms of Eilenberg and Steenrod. 
Proof. The proof is substantially the same in both cases. The validity of the first three axioms follows from $[11,4.1 .14,4.1 .15]$. That the Exactness Axiom is verified follows from [10, Chap. V Theorem 3.7] and the fact that the following sequence of morphisms of chain complexes is clearly exact:

$$
0 \longrightarrow \mathcal{F}_{*}(A) \stackrel{i_{*}}{\longrightarrow} \mathcal{F}_{*}(X) \stackrel{j_{*}}{\longrightarrow} \mathcal{F}_{*}(X, A) \longrightarrow 0
$$

whenever $(X, A)$ is an object of $\mathcal{A}$ and $i, j$ are the obvious inclusions. In order to check that the Homotopy Axiom holds we consider $f_{0}, f_{1}:(X, A) \rightarrow$ $(Y, B)$ and $h:(X \times[0,1], A \times[0,1]) \rightarrow(Y, B)$ such that $h(x, 0)=f_{0}(x)$ and $h(x, 1)=f_{1}(x), x \in X$. According to the homotopy formula [11, 4.1.9, 4.1.14] the homomorphisms

$$
D_{m}: \mathcal{F}_{m}(X, A) \rightarrow \mathcal{F}_{m+1}(Y, B):[T] \mapsto\left[h_{\#}([0,1] \times T)\right]
$$

define a chain homotopy of $f_{0 \#}$ into $f_{1 \#}$. Therefore $\mathbf{H}_{*}\left(f_{0}\right)=\mathbf{H}_{*}\left(f_{1}\right)$, [10, Chap. V Definition 4.1 and Theorem 4.4]. We turn to checking that the Excision Axiom holds (compare $[11,4.4 .1])$. Let $(X, A)$ be an object of $\mathcal{A}$ and $U \subset X$ be (relatively) open such that $\operatorname{Clos}_{X} U \subset \operatorname{Int}_{X} A$. Consider the composition of the homomorphisms

$$
\mathcal{F}_{m}(X \sim U) \stackrel{i_{\#}}{\longrightarrow} \mathcal{F}_{m}(X) \stackrel{p}{\longrightarrow} \frac{\mathcal{F}_{m}(X)}{\mathcal{F}_{m}(A)}
$$

We first show that $q=p \circ i_{\#}$ is onto. Define $u: \mathbb{R}^{n} \rightarrow \mathbb{R}$ by $u=\operatorname{dist}(\cdot, U)$, fix $T \in \mathcal{F}_{m}(X)$ and let $r_{0}>0$ be such that $u(x) \geq r_{0}$ whenever $x \in \operatorname{spt}(T) \sim A$. According to Lemma 3.5 there exist $r_{0} / 3<r<r_{0} / 2$ and $T_{u, r}^{-}, T_{u, r}^{+} \in \mathcal{F}_{m}(X)$ such that

$$
\operatorname{spt} T_{u, r}^{+} \subset X \sim U
$$

and

$$
\operatorname{spt}\left(T-T_{u, r}^{+}\right)=\operatorname{spt} T_{u, r}^{-} \subset A
$$

showing that $q$ is onto. We also trivially have that $\operatorname{ker} q=\mathcal{F}_{m}(X \sim A)$. Therefore (3.9) induces an ismorphism of the chain complexes $\mathcal{F}_{*}(X \sim$ $U, X \sim A)$ and $\mathcal{F}_{*}(X, A)$, so that $\mathbf{H}_{*}(X \sim U, A \sim A) \cong \mathbf{H}_{*}(X, A)$. Finally, the Dimension Axiom is checked by referring to [11, 4.2.14].

Remark 3.8. We show that the "coefficient group" of both theories is $\mathbb{Z}$ :

$$
\mathbf{H}_{0}(\{0\}) \cong \overline{\mathbf{H}}_{0}(\{0\}) \cong \mathbb{Z}
$$

Recall that $\mathcal{R}_{0}\left(\mathbb{R}^{n}\right)=\mathcal{P}_{0}\left(\mathbb{R}^{n}\right)=\mathbf{I}_{0}\left(\mathbb{R}^{n}\right)$ is the additive subgroup of $\mathcal{D}_{0}\left(\mathbb{R}^{n}\right)$ generated by $\boldsymbol{\delta}_{x}, x \in \mathbb{R}^{n}$. This clearly shows that $\overline{\mathbf{H}}_{0}(\{0\}) \cong \mathbb{Z}$. For settling 
the case of $\mathbf{H}_{0}(\{0\})$ we first recall that $\mathbf{I}_{0}\left(\mathbb{R}^{n}\right) \subsetneq \mathcal{F}_{0}\left(\mathbb{R}^{n}\right)$ (see Remark 3.6). Nevertheless we merely need to establish that

$$
\mathcal{F}_{0}(\{0\})=\left\{\nu \boldsymbol{\delta}_{0}: \nu \in \mathbb{Z}\right\}
$$

Let $T \in \mathcal{F}_{0}(\mathbb{R})$ be such that spt $T \subset\{0\}$ and choose $r>0$ and $S \in \mathcal{R}_{1}(\mathbb{R})$ such that spt $S \subset[-r, r]$ and

$$
\mathcal{F}_{[-r, r]}(T)=\mathbf{M}(T-\partial S)+\mathbf{M}(S) .
$$

Now for every $0<\varepsilon<1$ one has:

$$
T=\boldsymbol{\mu}_{\varepsilon \#} T=\boldsymbol{\mu}_{\varepsilon \#}(T-\partial S)+\partial \boldsymbol{\mu}_{\varepsilon \#} S,{ }^{7}
$$

therefore

$$
\begin{aligned}
\mathcal{F}_{[-r, r]}(T) & \leq \mathbf{M}\left(\boldsymbol{\mu}_{\varepsilon \#}(T-\partial S)\right)+\mathbf{M}\left(\boldsymbol{\mu}_{\varepsilon \#} S\right) \\
& =\mathbf{M}(T-\partial S)+\varepsilon \mathbf{M}(S)
\end{aligned}
$$

In view of (3.10), (3.11) implies that $S=0$, whence $\mathbf{M}(T)=\mathcal{F}_{[-r, r]}(T)<\infty$. In other words, $T$ is a distribution of order 0 supported in $\{0\}$, therefore $T=\alpha \boldsymbol{\delta}_{0}$ for some $\alpha \in \mathbb{R}$ (see $[17,6.25]$ ). Finally since $T$ is the weak limit of some $P_{j} \in \mathcal{P}_{0,[-r, r]}(\mathbb{R}), j=1,2, \ldots$, letting $\varphi \in \mathcal{D}^{0}(\mathbb{R})$ equal 1 on $[-r, r]$ we infer that $\alpha=\langle T, \varphi\rangle=\lim \left\langle P_{j}, \varphi\right\rangle \in \mathbb{Z}$.

In the remaining part of this section we will compare integral flat and integral rectifiable homology groups with Čech homology groups.

Lemma 3.9. Let $X \subset \mathbb{R}^{n}$ and let $U_{i} \subset X, i=1,2$, be (relatively) open. The following short sequence is exact:

$$
0 \rightarrow \mathcal{F}_{q}\left(U_{1} \cap U_{2}\right) \stackrel{\alpha_{2}}{\rightarrow} \mathcal{F}_{q}\left(U_{1}\right) \oplus \mathcal{F}_{q}\left(U_{2}\right) \stackrel{\alpha_{1}}{\rightarrow} \mathcal{F}_{q}\left(U_{1} \cup U_{2}\right) \rightarrow 0
$$

whenever $q \geq 0$, where $\alpha_{1}\left(T_{1}, T_{2}\right)=T_{1}+T_{2}$ and $\alpha_{2}(S)=(-S, S)$. The same holds with $\mathcal{F}_{q}$ replaced by $\mathbf{I}_{q}$.

Proof. It is clear that $\alpha_{2}$ is injective. Next if $T_{i} \in \mathcal{F}_{q}\left(U_{i}\right), i=1,2$, and $T_{1}+T_{2}=0$ then it is easily observed that $\operatorname{spt}\left(T_{1}\right) \cup \operatorname{spt}\left(T_{2}\right) \subset U_{1} \cap U_{2}$, whence $\left(T_{1}, T_{2}\right)=\alpha_{2}\left(-T_{1}\right)$. Finally, if $T \in \mathcal{F}_{q}\left(U_{1} \cup U_{2}\right)$ we start by choosing $r_{0}>0$ such that

$$
\operatorname{spt}(T) \cap\left\{x: \operatorname{dist}\left(x, \mathbb{R}^{n} \sim U_{2}\right) \leq r_{0}\right\} \subset U_{1} .
$$

\footnotetext{
${ }^{7} \boldsymbol{\mu}_{\varepsilon}(x)=\varepsilon x, x \in \mathbb{R}^{n}$.
} 
Next, applying Lemma 3.5 to $T$ and $u(x)=\operatorname{dist}\left(x, \mathbb{R}^{n} \sim U_{2}\right), x \in \mathbb{R}^{n}$, we select $0<r<r_{0}$ such that $T=T_{u, r}^{+}+T_{u, r}^{-}$. Clearly

$$
\operatorname{spt} T_{u, r}^{+} \subset U_{2},
$$

whereas

$$
\operatorname{spt} T_{u, r}^{-} \subset U_{1}
$$

follows from (3.12). Therefore $T=\alpha_{1}\left(T_{u, r}^{-}, T_{u, r}^{+}\right)$.

Corollary 3.10. Let $X \subset \mathbb{R}^{n}$ and $q=0,1,2, \ldots$ Then the functor $\mathcal{F}_{q}$ : $\mathrm{O}(X) \rightarrow \operatorname{Mod}_{\mathbb{Z}}: U \mapsto \mathcal{F}_{q}(U)$ is a cosheaf. The same holds for the functor $\mathbf{I}_{q}: \mathrm{O}(X) \rightarrow \operatorname{Mod}_{\mathbb{Z}}: U \mapsto \mathbf{I}_{q}(U)$.

Proof. This is an application of Proposition 2.10. Hypothesis (1) of that Proposition is satisfied according to Lemma 3.9 whereas hypothesis (2) is checked as follows. Assume that $\left\{U_{i}: i \in I\right\}$ is a countable family of (relatively) open subsets of $X$ directed upwards by inclusion and let $T \in$ $\mathcal{F}_{q}(X)$. Since $\operatorname{spt} T$ is compact there exists $i \in I$ such that $\operatorname{spt} T \subset U_{i}$, i.e. $T \in \mathcal{F}_{q}\left(U_{i}\right)$. This shows that the natural homomorphism

$$
\lim _{\overrightarrow{i \in I}} \mathcal{F}_{q}\left(U_{i}\right) \longrightarrow \mathcal{F}_{q}(X)
$$

is an isomorphism.

Definition 3.11. Let $X \subset \mathbb{R}^{n}$ and $m \geq 0$. We say that $X$ is $(\mathbf{H}, m)$ locally connected (resp. $(\overline{\mathbf{H}}, m)$ locally connected) if the following condition holds. For every $x \in X$ and every open neighborhood $U$ of $x$ there exists an open neighborhood $U^{\prime} \subset U$ of $x$ such that the homomorphism induced by inclusion in reduced integral flat homology (resp. integral rectifiable homology)

$$
\mathcal{F}_{q}\left(U^{\prime}\right) \rightarrow \mathcal{F}_{q}(U) \quad\left(\operatorname{resp} . \mathbf{I}_{q}\left(U^{\prime}\right) \rightarrow \mathbf{I}_{q}(U)\right)
$$

is trivial for $q=0, \ldots, m+1$.

Example 3.12. It is most obvious that a Lipschitzian manifold $X \subset \mathbb{R}^{n}$ is $(\mathbf{H}, m)$ and $(\overline{\mathbf{H}}, m)$ locally connected for each $m \geq 0$ : each $x \in X$ admits arbitrarily small neighborhoods which are contractible in the Lipschitz category.

Definition 3.13. Let $m \geq 0$. We denote by $\mathcal{A}^{L C, \mathbf{H}, m}\left(\operatorname{resp} . \mathcal{A}^{L C, \overline{\mathbf{H}}, m}\right)$ the category whose objects are the $(\mathbf{H}, m)$ (resp. $(\overline{\mathbf{H}}, m))$ locally connected subsets $X \subset \mathbb{R}^{n}$ of some Euclidean space, together with their locally Lipschitzian maps. 
The proof of the following result is analogous to that of Theorem 2.15.

Theorem 3.14. Let $0 \leq q \leq m$ be integers. The functors

$$
\mathbf{H}_{q}: \mathcal{A}^{L C, \mathbf{H}, m} \rightarrow \operatorname{Mod}_{\mathbb{Z}} \quad \text { integral flat homology }
$$

and

$$
\check{H}_{q}: \mathcal{A}^{L C, \mathbf{H}, m} \rightarrow \operatorname{Mod}_{\mathbb{Z}} \quad \text { Cech homology }
$$

are naturally equivalent. The same holds with $\mathbf{H}$ replaced by $\overline{\mathbf{H}}$.

\subsection{Counter-examples}

We now provide examples to the effect that for each distinct theories $\mathfrak{h}, \mathfrak{H} \in$ $\{H, \check{H}, \mathbf{H}, \overline{\mathbf{H}}\}$ with $\{\mathfrak{h}, \mathfrak{H}\} \neq\{H, \check{H}\}$ there is not necessarily a monomorphism $\mathfrak{h}_{*}(X) \hookrightarrow \mathfrak{H}_{*}(X)$ whenever $X$ is a closed subset of some Euclidean space. None of the closed sets $X$ appearing in the following examples is a Lipschitz neighborhood retract.

Example 3.15. Here we consider a von Koch curve $X \subset \mathbb{R}^{2}$, which is biLipschitzian equivalent to $[0,1]$ equipped with the metric $d(x, y)=|x-y|^{s}$, $x, y \in[0,1]$, where $s=\frac{\log 3}{\log 4}$ (see for instance [1]). Then of course $X$ is continuously contractible so that $H_{0}(X ; \mathbb{Z}) \cong \check{H}_{0}(X ; \mathbb{Z}) \cong \mathbb{Z}$. On the other hand if $a, b \in X, a \neq b$, then there is no $T \in \mathbf{I}_{1}(X)$ such that $\partial T=\boldsymbol{\delta}_{b}-\boldsymbol{\delta}_{a}$. In fact every indecomposable (see $[11,4.2 .25]) T \in \mathbf{I}_{1}\left(\mathbb{R}^{2}\right)$ such that $\operatorname{spt} T \subset X$ is necessarily equal to zero. This is because $T=f_{\#}\left(\mathbf{E}^{1}\llcorner[0, \mathbf{M}(T)])\right.$ for some one-to-one Lipschitzian function $f: \mathbb{R} \rightarrow \mathbb{R}^{2}$, therefore there is a Lipschitzian bijection $([0, \mathbf{M}(T)],|\cdot|) \rightarrow\left(\left[t_{1}, t_{2}\right], d\right)$, implying its image has finite length, whence $t_{2}=t_{1}$ and in turn $\mathbf{M}(T)=0$. We conclude that $\mathbf{I}_{1}(X)=\{0\}$ and $\overline{\mathbf{H}}_{0}(X) \cong \oplus_{x \in X} \mathbb{Z}$. One shows that $\mathbf{H}_{0}(X) \cong \mathbb{Z}$ (see the next Example for a similar argument).

Example 3.16. We now let $X \subset \mathbb{R}^{2}$ be the topological boundary of a von Koch snowflake $Z \subset \mathbb{R}^{2}$. Then $X$ is homeomorphic to $\mathbf{S}^{1}$ so that $H_{1}(X ; \mathbb{Z}) \cong \check{H}_{1}(X ; \mathbb{Z}) \cong \mathbb{Z}$. It follows from Example 3.15 that $\overline{\mathbf{H}}_{1}(X) \cong\{0\}$. Next we shall show that $\mathbf{H}_{1}(X) \cong \mathbb{Z}$. Notice that $S=\mathbf{E}^{2}\left\llcorner Z \in \mathcal{R}_{2}\left(\mathbb{R}^{2}\right)\right.$, whence $T=\partial S \in \mathcal{F}_{1}\left(\mathbb{R}^{2}\right)$. Moreover $T \neq 0$, for the contrary would imply $S=0$ according to the constancy Theorem [11, 4.1.7], in contradiction with $\mathcal{L}^{2}(Z)>0$. Furthermore if $T^{\prime} \in \mathcal{F}_{1}(X)$ and $\partial T^{\prime}=0$ then $T^{\prime}=\nu T$ for some $\nu \in \mathbb{Z}$. Indeed, letting $S^{\prime}=\boldsymbol{\delta}_{a} \mathbb{W}^{\prime}$ (for $a \in \operatorname{Int} Z$ ) one infers that spt $\partial\left(S-S^{\prime}\right) \cap \operatorname{Int} Z=\emptyset$ so that $S-S^{\prime}=c \mathbf{E}^{2}\llcorner\operatorname{Int} Z$ for some $c \in \mathbb{R}$, again according to the constancy Theorem. Clearly $c \in \mathbb{Z}$. This shows that $\mathcal{F}_{1}(X) \cap\{T: \partial T=0\} \cong \mathbb{Z}$. Finally $\mathcal{F}_{2}(X)=\{0\}$ because $\mathcal{L}^{2}(X)=0$ (recall [11, 4.1.20]). 
Example 3.17. Let $f(x)=\left(x,|x| \sin |x|^{-1}\right) \in \mathbb{R}^{3}, x \in \mathbb{R}^{2} \sim\{0\}$, and $f(0)=0$. The set $X=f(\mathbf{B}(0,1))$ is $\left(\mathcal{H}^{2}, 2\right)$ rectifiable (in particular $\left.\mathcal{H}^{2}(X)<\infty\right)$. It is also clearly continuously contractible, therefore $H_{0}(X ; \mathbb{Z})$ $\cong \check{H}_{0}(X ; \mathbb{Z}) \cong \mathbb{Z}$. If $a, b \in X \sim\{0\}, a \neq b$, then it is easy to see that there exists $T \in \mathbf{I}_{1}(X)$ such that $\partial T=\boldsymbol{\delta}_{b}-\boldsymbol{\delta}_{a}$. On the other hand, if $b \in X \sim\{0\}$ then there is no $T \in \mathbf{I}_{1}(X)$ such that $\partial T=\boldsymbol{\delta}_{b}-\boldsymbol{\delta}_{0}$. For if such $T$ existed, we could assume $T$ to be of least mass ([11, 4.2.17(2)]), and in turn a simple argument would imply $T\left\llcorner\left\{x:\left|\boldsymbol{p}_{(1,2)}(x)\right| \geq r\right\}\right.$ equals $T_{r}=f_{\#}\left[r \boldsymbol{p}_{(1,2)}(b)\left|\boldsymbol{p}_{(1,2)}(b)\right|^{-1}, \boldsymbol{p}_{(1,2)}(b)\right]$. This, however, leads to a contradiction since $\mathbf{M}\left(T_{r}\right) \rightarrow \infty$ as $r \rightarrow 0^{+}$. We conclude $\overline{\mathbf{H}}_{0}(X) \cong \mathbb{Z} \oplus \mathbb{Z}$. Finally one shows (like in Example 3.16) there is $T \in \mathcal{F}_{1}(X)$ such that $\partial T=\boldsymbol{\delta}_{b}-\boldsymbol{\delta}_{0}$. Therefore $\mathbf{H}_{0}(X) \cong \mathbb{Z}$.

Example 3.18. Let $r(\theta)=\left(1+\frac{\theta}{2 \pi}\right)^{-1 / 2}, \theta \geq 0$, and define the set $X=\mathbb{R}^{2} \cap$ $\{(r(\theta) \cos \theta, r(\theta) \sin \theta): \theta \geq 0\} \cup\{0\}$. Then $X$ is continuously contractible so that $H_{0}(X ; \mathbb{Z}) \cong \check{H}_{0}(X ; \mathbb{Z}) \cong \mathbb{Z}$. If $a, b \in X \sim\{0\}, a \neq b$, then clearly there exists $T \in \mathbf{I}_{1}(X)$ such that $\partial T=\boldsymbol{\delta}_{b}-\boldsymbol{\delta}_{a}$. If $b \in X \sim\{0\}$ we claim there is no $T \in \mathcal{F}_{1}(X)$ such that $\partial T=\boldsymbol{\delta}_{b}-\boldsymbol{\delta}_{0}$. For if such $T$ existed we would infer that $S=T+[b, 0] \in \mathcal{F}_{1}\left(\mathbb{R}^{2}\right)$ and $\partial S=0$, implying that $\boldsymbol{\delta}_{0} \times S \in \mathcal{R}_{2}\left(\mathbb{R}^{2}\right)$; however the slow rate of decrease of $r(\theta)$ easily implies that such $\boldsymbol{\delta}_{0} \times \times S$ cannot have finite mass. We conclude $\mathbf{H}_{0}(X) \cong \overline{\mathbf{H}}_{0}(X) \cong \mathbb{Z} \oplus \mathbb{Z}$.

\section{Appendix A: Vocabulary from category theory}

In this Appendix we review the vocabulary borrowed from category theory which makes cleaner the exposition of the core paper. We have chosen $[2,3]$ as a reference for Definitions. Here is a short list of basic notions together with a link to their Definition: for a category see [2, 1.2.1], for a functor see $[2,1.2 .2]$, for a natural transformation between functors see [2, 1.3.1], for a monomorphism see [2, 1.7.1], for an epimorphism see [2, 1.8.1], for an isomorpshism see $[2,1.9 .1]$. Notice that we will only consider covariant functors and we will call these simply functors. A natural equivalence $\nu$ between two functors $F, G: \mathrm{C} \rightarrow \mathrm{D}$ is a natural transformation such that for each object $A$ of $C$ the morphism $\nu_{A}: F(A) \rightarrow G(A)$ is an isomorphism.

We now review some of the categories used in the paper. We let $A b$ denote the catgeory of abelian groups and their homomorphisms. We denote by $A b_{c}$ the category of compact topological abelian groups and their continuous homomorphisms. Whenever $R$ is a ring, we let $\operatorname{Mod}_{R}$ be the category of $R$ modules and their homomorphisms. Top and Comp denote respectively the categories of topological spaces and compact Hausdorff topological spaces together with their continuous maps. We denote by $\mathrm{Top}_{2}$, and similarly 
Comp $_{2}$, the category of pairs $(X, A)$ of objects of Top (respectively Comp) such that $A \subset X$, the morphisms $(X, A) \rightarrow(Y, B)$ being continuous maps $f$ : $X \rightarrow Y$ such that $f(A) \subset B$. When $X$ is a topological space, we let $\mathrm{O}(X)$ be the category whose objects are the open sets of $X$ and whose morphisms are defined as follows: there is exactly one morphism between $U$ and $V$ if $U \subset V$ and none otherwise. This is a particular case of the category $\mathrm{J}$ associated with a set $J$ and a partial order $\preceq$ on $J$ : the objects of $J$ are the elements of $J$ and there is exactly one morphism between $j_{1}$ and $j_{2}$ whenever $j_{1} \preceq j_{2}$, otherwise there is none. We also refer to [10, Chapter I, Definition p. 5] for the Definition of an admissible category for homology theory.

An inverse system in a category $\mathrm{C}$ consists of the following data: a directed set $(J, \preceq)$ together with a functor $F: \mathrm{J} \rightarrow \mathrm{C}$. We will denote such an inverse system by $(F, J)$. For instance an inverse system of abelian groups indexed by $J, G: \mathrm{J} \rightarrow \mathrm{Ab}$, consists of an indexed family of abelian groups $G_{j}=G(j), j \in J$, together with homomorphisms $\pi^{j_{1}, j_{2}}: G_{j_{2}} \rightarrow G_{j_{1}}$, $\pi^{j_{2}, j_{1}}=G\left(j_{1} \rightarrow j_{2}\right)$, corresponding to each pair $j_{1}, j_{2} \in J$ such that $j_{1} \preceq j_{2}$. These data are subject to the following conditions: $\pi^{j, j}=\mathrm{id}_{G_{j}}$ for every $j \in J$ and $\pi^{j_{1}, j_{2}} \circ \pi^{j_{2}, j_{3}}=\pi^{j_{1}, j_{3}}$ for every triple $j_{1}, j_{2}, j_{3} \in J$ such that $j_{1} \preceq j_{2} \preceq j_{3}$. A morphism of inverse systems $\Phi:(F, J) \rightarrow(G, \mathrm{~K})$ consists of the following data: an order preserving map $\varphi: K \rightarrow J$ and, for each $k \in K$, a morphism $\phi_{k}: F(\varphi(k)) \rightarrow G(k)$. These data are subject to the following requirement: whenever $k_{1}, k_{2} \in K$ and $k_{1} \preceq k_{2}$ the following diagram is commutative:

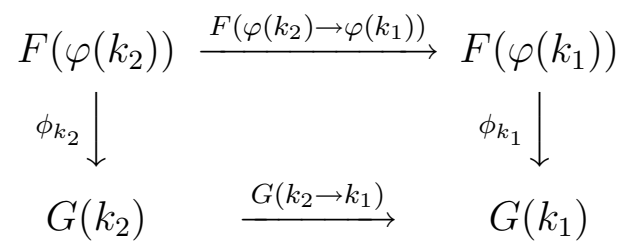

It is most obvious how to define the composition of two such morphisms of inverse systems. This provides us with a new category $\operatorname{Inv}(\mathrm{C})$ : that of inverse systems in $\mathrm{C}$ and their morphisms.

We now define inverse cones and inverse limits as in [2, 2.6.1,2.6.2] where these are called cones and limits. An inverse cone on an inverse system $(F, \mathrm{~J})$ consists of an object $C$ of $C$ together with morphisms $p_{j}: C \rightarrow F(j), j \in J$, verifying the following condition: $F\left(j_{2} \rightarrow j_{1}\right) \circ p_{j_{2}}=p_{j_{1}}$ whenever $j_{1}, j_{2} \in J$ and $j_{1} \preceq j_{2}$. An inverse limit of an inverse system $(F, J)$ is an inverse cone $\left(L,\left(p_{j}\right)_{j \in J}\right)$ on $(F, J)$ such that for every other inverse cone $\left(M,\left(q_{j}\right)_{j \in J}\right)$ on $(F, \mathrm{~J})$ there exists a unique morphism $m: M \rightarrow L$ such that $q_{j}=p_{j} \circ m$ for each $j \in J$. If an inverse limit $\left(L,\left(p_{j}\right)_{j \in J}\right)$ exists then the object $L$ is uniquely determined up to isomorphism. Inverse limits exist in the following 
categories: $\mathrm{Ab}, \mathrm{Ab}_{\mathrm{c}}$, Top, Comp, $\mathrm{Top}_{2}, \mathrm{Comp}_{2}$. In fact one can choose

$$
\begin{aligned}
L=\prod_{j \in J} F(j) \cap\left\{\left(x_{j}\right)_{j \in J}: F\left(j_{2}\right.\right. & \left.\rightarrow j_{1}\right)\left(x_{j_{2}}\right)=x_{j_{1}} \\
& \text { whenever } \left.j_{1}, j_{2} \in J \text { and } j_{1} \preceq j_{2}\right\},
\end{aligned}
$$

and for $p_{j}$ the restriction of the projection on the $j^{\text {th }}$ factor. This particular $\operatorname{limit} L$ will be denoted $\lim (F, \mathrm{~J})$ or

$$
\lim _{j \in J} F(j)
$$

Now if $(\varphi, \phi):(F, \mathrm{~J}) \rightarrow(G, \mathrm{~K})$ is a morphism of inverse systems then we define

$$
\lim _{\leftarrow}(\varphi, \phi): \lim _{\leftarrow}(F, \mathrm{~J}) \rightarrow \underset{\leftarrow}{\lim }(G, \mathrm{~K})
$$

to be the unique morphism associated with the inverse cone $\left(\lim _{\leftarrow}(F, J),\left(\phi_{k} \circ\right.\right.$ $\left.\left.p_{\varphi(k)}\right)_{k \in K}\right)$ on $(G, \mathrm{~K})$. It is an easy matter to check that this defines a functor (see for instance [10, Chapter VIII, Theorem 3.14] for some inspiration):

$$
\lim _{\leftarrow}: \operatorname{lnv}(C) \rightarrow C
$$

whenever $C$ is a complete category (see $[2,2.7 .2]$ ). It is also an exercise to show that if $J^{\prime} \subset J$ is cofinal then the objects $\lim (F, \mathrm{~J})$ and $\lim \left(F, \mathrm{~J}^{\prime}\right)$ are isomorphic in C, [10, Chapter VIII, Corollary 3.16].

\section{Appendix B: Hausdorff measures and rectifiability}

Let $m \geq 0$ be a real number, $\delta>0$ and $X \subset \mathbb{R}^{n}$. We define

$$
\begin{array}{r}
\mathcal{H}_{\delta}^{m}(X)=\inf \left\{\sum_{i \in I} \boldsymbol{\alpha}(m) 2^{-m}\left(\operatorname{diam} X_{i}\right)^{m}: X_{i} \subset \mathbb{R}^{n}, \operatorname{diam} X_{i} \leq \delta, i \in I,\right. \\
\text { and } \left.X \subset \cup_{i \in I} X_{i}\right\}
\end{array}
$$

where $\boldsymbol{\alpha}(m)>0$ is a normalizing constant. Next we define

$$
\mathcal{H}^{m}(X)=\sup \left\{\mathcal{H}_{\delta}^{m}(X): \delta>0\right\}
$$

This is the $m$ dimensional Hausdorff measure of $X$. The set function $\mathcal{H}^{m}$ is an outer measure, Borel subsets of $\mathbb{R}^{n}$ are $\mathcal{H}^{m}$ measurable, $\mathcal{H}^{n}$ is the Lebesgue measure on $\mathbb{R}^{n}, \mathcal{H}^{0}$ is the counting measure and if $0<m<n$ is an integer then $\mathcal{H}^{m}(M)$ coincides with the "classical $m$ dimensional area" whenever $M \subset \mathbb{R}^{n}$ is an $m$ dimensional $C^{1}$ submanifold. 
For the remainder of this Appendix we will assume that $0 \leq m \leq n$ is an integer. A set $X \subset \mathbb{R}^{n}$ is called countably $\left(\mathcal{H}^{m}, m\right)$ rectifiable if there are subsets $Z_{j} \subset \mathbb{R}^{m}$ and Lipschitzian functions $f_{j}: Z_{j} \rightarrow \mathbb{R}^{n}, j=1,2, \ldots$, such that $\mathcal{H}^{m}\left(X \sim \cup_{j=1}^{\infty} f_{j}\left(Z_{j}\right)\right)=0$. We say that $X$ is $\left(\mathcal{H}^{m}, m\right)$ rectifiable if it is countably $\left(\mathcal{H}^{m}, m\right)$ rectifiable and $\mathcal{H}^{m}(X)<\infty$. In order to state several characterizations of rectifiability we need some more Definitions. Radon measures on $\mathbb{R}^{n}$ are defined for instance in [14]. If $X \subset \mathbb{R}^{n}$ is $\mathcal{H}^{m}$ measurable and if $\mathcal{H}^{m}(C \cap X)<\infty$ for every compact $C \subset \mathbb{R}^{n}$ then the measure $\mathcal{H}^{m}\llcorner X$ defined as follows is Radon:

$$
\left(\mathcal{H}^{m}\llcorner X)(B)=\mathcal{H}^{m}(X \cap B), B \subset \mathbb{R}^{n} .\right.
$$

Given a Radon measure $\mu$ on $\mathbb{R}^{n}$ we let

$$
\Theta_{*}^{m}(\mu, x)=\liminf _{r \rightarrow 0} \frac{\mu(\mathbf{B}(x, r))}{\boldsymbol{\alpha}(m) r^{m}}
$$

and

$$
\Theta^{m *}(\mu, x)=\limsup _{r \rightarrow 0} \frac{\mu(\mathbf{B}(x, r))}{\boldsymbol{\alpha}(m) r^{m}}
$$

whenever $x \in \mathbb{R}^{n}$ and $r>0$. We also let $T_{x, r}: \mathbb{R}^{n} \rightarrow \mathbb{R}^{n}: y \mapsto(y-x) / r$ and we say that a Radon measure $\nu$ is $m$ tangent to $\mu$ at $x$ if there exists a decreasing sequence $r_{j}, j=1,2, \ldots$, tending to 0 such that

$$
r_{j}^{-m} T_{x, r_{j} \#} \mu \rightarrow \nu \text { as } j \rightarrow \infty
$$

weakly in the sense of Radon measures. We let $\mathbf{G}(n, m)$ denote the Grassmannian of $m$ dimensional vectorsubspaces of $\mathbb{R}^{n}$ and we say that a Radon measure $\nu$ is $m$ flat if $\nu=\mathcal{H}^{m}\llcorner W$ for some $W \in \mathbf{G}(n, m)$.

Theorem 3.19. Let $X \subset \mathbb{R}^{n}$ be $\mathcal{H}^{m}$ measurable and assume that $\mathcal{H}^{m}(X \cap$ $C)<\infty$ for every compact $C \subset \mathbb{R}^{n}$ and that $\Theta_{*}^{m}\left(\mathcal{H}^{m}\llcorner X, x)>0\right.$ for $\mathcal{H}^{m}\llcorner X$ almost every $x \in \mathbb{R}^{n}$. The following conditions are equivalent:

(A) $X$ is countably $\left(\mathcal{H}^{m}, m\right)$ rectifiable;

(B) There are $m$ dimensional submanifolds of class $C^{1}, M_{j} \subset \mathbb{R}^{n}, j=$ $1,2, \ldots$, such that $\mathcal{H}^{m}\left(X \sim \cup_{j=1}^{\infty} M_{j}\right)=0$;

(C) For $\mathcal{H}^{m}\left\llcorner X\right.$ almost every $x \in \mathbb{R}^{n}, \mathcal{H}^{m}\llcorner X$ has a unique $m$ tangent measure at $x$ and it is $m$ flat;

(D) For $\mathcal{H}^{m}\left\llcorner X\right.$ almost every $x \in \mathbb{R}^{n}$, all $m$ tangent measures to $\mathcal{H}^{m}\llcorner X$ at $x$ are $m$ flat;

(E) For $\mathcal{H}^{m}\left\llcorner X\right.$ almost every $x \in \mathbb{R}^{n}$,

$$
\Theta^{m *}\left(\mathcal{H}^{m}\llcorner X, x)=\Theta_{*}^{m}\left(\mathcal{H}^{m}\llcorner X, x)=1 .\right.\right.
$$


We now briefly mention the major ingredients needed for proving this Theorem. That (A) implies (B) follows from H. Whitney's extension Theorem, $[11,3.1 .14]$, and the reverse implication is obvious. That (A) or (B) implies (C) is a consequence of the area formula, $[11,3.2 .3]$. It is clear that (C) implies (D); (E) follows from (D) and a straightforward calculation. The proof that (D) implies (A) can be found in $[14,16.5]$. Finally the Theorem that (E) implies (D) is due to P. Mattila and can be found e.g. in $[14,17.3,17.6]$ (D. Preiss proved later a stronger result, [15]). In case $X$ verifies the conditions of the Theorem and $\nu$ is the unique $m$ tangent measure to $\mathcal{H}^{m}\llcorner X$ at $x$ and is $m$ flat, we call approximate tangent space of $X$ at $x$ the $m$ dimensional subspace $W \in \mathbf{G}(n, m)$ such that $\nu=\mathcal{H}^{m}\llcorner W$.

\section{References}

[1] Assouad, P.: Plongements Lipschitziens dans $\mathbb{R}^{n}$. Bull. Soc. Math. France 111 (1983), 429-448.

[2] Borceux, F.: Handbook of categorical algebra. 1. Basic category theory. Encyclopedia of Mathematics and its Applications 50. Cambridge University Press, Cambridge, 1994.

[3] Borceux, F.: Handbook of categorical algebra. 2. Categories and structures. Encyclopedia of Mathematics and its Applications 51. Cambridge University Press, Cambridge, 1994.

[4] Bott, R. And Tu, L. W.: Differential forms in algebraic topology. Graduate Texts in Mathematics 82. Springer-Verlag, New York-Berlin, 1982.

[5] Bredon, G. E.: Cosheaves and homology. Pacific J. Math. 25 (1968), 1-32.

[6] De Pauw, Th.: Size minimizing surfaces with integral coefficients. Forthcoming.

[7] De Pauw, Th.: Approximating compact rectifiable sets in measure and Hausdorff distance by polyhedral Lipschitzian images. Forthcoming.

[8] De Rham, G.: Variétés différentiables: formes, courants, formes harmoniques. Hermann, Paris, 1960.

[9] Dugundji, J.: Topology. Allyn and Bacon, Inc., 1966.

[10] Eilenberg, S. And Steenrod, N.: Foundations of algebraic topology. Princeton University Press, Princeton, New Jersey, 1952.

[11] Federer, H.: Geometric measure theory. Die Grundlehren der mathematischen Wissenschaften 153. Springer-Verlag, New York, 1969.

[12] Federer, H. and Fleming, W. H.: Normal and integral currents. Ann. of Math. (2) 72 (1960), 458-520.

[13] Mardešić, S.: Comparison of singular and Čech homology in locally connected spaces. Michigan Math. J. 6 (1959), 151-166. 
[14] Mattila, P.: Geometry of sets and measures in Euclidean spaces. Fractals and rectifiability. Cambridge Studies in Advanced Mathematics 44. Cambridge University Press, Cambridge, 1995.

[15] Preiss, D.: Geometry of measures in $\mathbb{R}^{n}$ : distribution, rectifiability, and densities. Ann. of Math. (2) 125 (1987), 537-643.

[16] Reifenberg, E. R.: Solution of the Plateau Problem for $m$-dimensional surfaces of varying topological type. Acta Math. 104 (1960), 1-92.

[17] Rudin, W.: Functional analysis. McGraw-Hill Series in Higher Mathematics. McGraw-Hill Book Co., New York-Düsseldorf-Johannesburg, 1973.

[18] Sullivan, D.: Hyperbolic geometry and homeomorphisms. In Geometric topology (Proc. Georgia Topology Conf., Athens, Ga., 1977), 543-555. Academic Press, New York-London, 1979.

[19] Weil, A.: Sur les théorèmes de De Rham. Comment. Math. Helv. 26 (1952), 119-145.

[20] Whitney, H.: Geometric integration theory. Princeton Mathematical Series 21. Princeton University Press, 1957.

Recibido: 15 de febrero de 2005

Revisado: 13 de octubre de 2005

Thierry De Pauw

Université catholique de Louvain

Département de mathématiques

2 chemin du cyclotron, 1348

Louvain-la-Neuve, Belgique depauw@math.ucl.ac.be

The author is a chercheur qualifié of the Fonds National de la Recherche Scientifique, Belgium. 